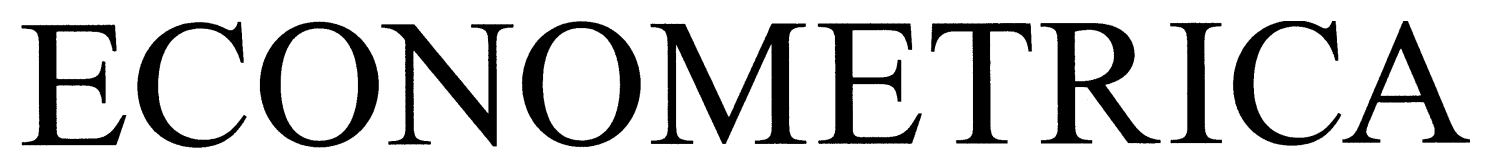

JOURNAL OF THE ECONOMETRIC SOCIETY

An International Society for the Advancement of Economic

Theory in its Relation to Statistics and Mathematics

http://www.econometricsociety.org/

Econometrica, Vol. 85, No. 3 (May, 2017), 693-734

EARNINGS AND CONSUMPTION DYNAMICS: A NONLINEAR PANEL

DATA FRAMEWORK

MANUEL ARELLANO

CEMFI, Madrid 28014, Spain

RICHARD BLUNDELL

University College London, London WC1E 6BT, U.K. and Institute for Fiscal Studies,

London WC1E 7AE, U.K.

STÉPHANE BONHOMME

University of Chicago, Chicago, IL 60637, U.S.A.

The copyright to this Article is held by the Econometric Society. It may be downloaded, printed and reproduced only for educational or research purposes, including use in course packs. No downloading or copying may be done for any commercial purpose without the explicit permission of the Econometric Society. For such commercial purposes contact the Office of the Econometric Society (contact information may be found at the website http://www.econometricsociety.org or in the back cover of Econometrica). This statement must be included on all copies of this Article that are made available electronically or in any other format. 


\title{
EARNINGS AND CONSUMPTION DYNAMICS: A NONLINEAR PANEL DATA FRAMEWORK
}

\author{
By Manuel Arellano, Richard Blundell, And Stéphane Bonhomme ${ }^{1}$
}

\begin{abstract}
We develop a new quantile-based panel data framework to study the nature of income persistence and the transmission of income shocks to consumption. Log-earnings are the sum of a general Markovian persistent component and a transitory innovation. The persistence of past shocks to earnings is allowed to vary according to the size and sign of the current shock. Consumption is modeled as an age-dependent nonlinear function of assets, unobservable tastes, and the two earnings components. We establish the nonparametric identification of the nonlinear earnings process and of the consumption policy rule. Exploiting the enhanced consumption and asset data in recent waves of the Panel Study of Income Dynamics, we find that the earnings process features nonlinear persistence and conditional skewness. We confirm these results using population register data from Norway. We then show that the impact of earnings shocks varies substantially across earnings histories, and that this nonlinearity drives heterogeneous consumption responses. The framework provides new empirical measures of partial insurance in which the transmission of income shocks to consumption varies systematically with assets, the level of the shock, and the history of past shocks.
\end{abstract}

KEYWORDS: Earnings dynamics, consumption, nonlinear persistence, partial insurance, panel data, quantile regression, latent variables.

\section{INTRODUCTION}

CONSUMPTION DECISIONS AND EARNINGS DYNAMICS are intricately linked. Together with the net value of assets, the size and durability of any income shock dictates how much consumption will need to adjust to ensure a reasonable standard of living in future periods of the life-cycle. ${ }^{2}$ Understanding the persistence of earnings is therefore of key interest not only because it affects the permanent or transitory nature of inequality, but also because it drives much of the variation in consumption. ${ }^{3}$ The precise nature of labor income dynamics and the distribution of idiosyncratic shocks also plays a central role in the design of optimal social insurance and taxation. ${ }^{4}$ This paper proposes a new nonlinear framework to study the persistence of earnings and the impact of earnings shocks on consumption.

\footnotetext{
${ }^{1}$ This paper was the basis for Arellano's Presidential Address to the Econometric Society in 2014. We are grateful to the co-editor and three anonymous referees for detailed comments. We also thank participants in regional meetings and seminars, especially Xiaohong Chen, Mariacristina De Nardi, Jordi Gali, Fatih Guvenen, Lars Hansen, Jim Heckman, Yingyao Hu, Josep Pijoan, Enrique Sentana, and Kjetil Storesletten for their comments. We are particularly grateful to Luigi Pistaferri and Itay Saporta-Eksten for help with the PSID data, Magne Mogstad and Michael Graber for providing the estimations using the Norwegian population register data as part of the project on 'Labour Income Dynamics and the Insurance from Taxes, Transfers and the Family', and Ran Gu and Raffaele Saggio for excellent research assistance. Arellano acknowledges research funding from the Ministerio de Economía y Competitividad, Grant ECO2011-26342. Blundell would like to thank the ESRC Centre CPP at IFS and the ERC MicroConLab project for financial assistance. Bonhomme acknowledges support from the European Research Council/ERC Grant agreement $n^{0} 263107$.

${ }^{2}$ See, for example, Jappelli and Pistaferri (2010) and references therein.

${ }^{3}$ See Deaton and Paxson (1994) for key initial insights on consumption inequality, and the subsequent literature reviewed in Blundell (2014).

${ }^{4}$ Golosov and Tsyvinski (2015) provided a recent review. In a dynamic Mirrlees tax design setting, optimal labor distortions for unexpectedly high shocks are determined mainly by the need to provide intertemporal insurance. Golosov, Troshkin, and Tsyvinski (2016) showed that deviations from log normality can have serious repercussions for optimal capital and labor taxation.
} 
With some notable exceptions (see the discussion and references in Meghir and Pistaferri (2011)), the literature on earnings dynamics has focused on linear models. The random-walk permanent/transitory model is a popular example (Abowd and Card (1989)). Linear models have the property that all shocks are associated with the same persistence, irrespective of the household's earnings history. Linearity is a convenient assumption, as it allows one to study identification and estimation using standard covariance techniques. However, by definition, linear models rule out nonlinear transmission of shocks, while nonlinearities in income dynamics are likely to have a first-order impact on consumption and saving choices.

The existing literature on earnings shocks and consumption follows two main approaches. One approach is to take a stand on the precise mechanisms that households use to smooth consumption, for example saving and borrowing or labor supply, and to take a fully-specified life-cycle model to the data; see Gourinchas and Parker (2002), Guvenen and Smith (2014), or Kaplan and Violante (2014), for example. Except in very special cases (as in Hall and Mishkin (1982)), the consumption function is generally a complex nonlinear function of earnings components. ${ }^{5}$ Another approach is to estimate the degree of "partial insurance" from the data without precisely specifying the insurance mechanisms; see Blundell, Pistaferri, and Preston (2008), for example. Linear approximations to optimality conditions from the optimization problem deliver tractable estimating equations. However, linear approximations may not always be accurate (Kaplan and Violante (2010)). Moreover, some aspects of consumption smoothing, such as precautionary savings or asset accumulation in the presence of borrowing constraints and nonlinear persistence, are complex in nature, making a linear framework less attractive. In this paper, we develop a comprehensive new approach to study the nonlinear relationship between shocks to household earnings and consumption over the life-cycle.

Our first contribution is to specify and estimate a nonlinear earnings process. In this framework, log-earnings are the sum of a general Markovian persistent component and a transitory innovation. Our interest mainly centers on the conditional distribution of the persistent component given its past. This is a comprehensive measure of the earnings risk faced by households. Conditional (or predictive) earnings distributions are a main feature of many models of consumption responses to income shocks, as in Kaplan and Violante (2014), for example, and also play a central role in the optimal design of social insurance, as in Golosov, Troshkin, and Tsyvinski (2016). Using quantile methods on both U.S. and Norwegian data, we show that the conditional distribution of the persistent component of earnings exhibits important asymmetries. Our setup provides a tractable framework for incorporating such properties into structural decision models.

Our modeling approach to earnings dynamics captures the intuition that, unlike in linear models, different shocks may be associated with different persistence. The notion of persistence we propose is one of persistence of histories. In a Markovian setup, this is conveniently summarized using a derivative effect which measures by how much earnings at period $t$ vary with the earnings component at $t-1$, when hit by a particular shock at time $t$. This approach provides a new dimension of persistence where the impact of past shocks on current earnings may be altered by the size and sign of new shocks. For example, our framework allows for "unusual" shocks to wipe out the memory of past shocks.

Allowing for nonlinear persistence, and more generally for flexible models of conditional earnings distributions given past earnings, has both theoretical and empirical appeal. The main features of our nonlinear framework for earnings dynamics relate directly

\footnotetext{
${ }^{5}$ Interesting recent exceptions are Heathcote, Storesletten, and Violante (2014) and the semi-structural approach in Alan, Browning, and Ejrnaes (2014).
} 
to existing structural labor market models. Consider a worker who accumulates occupational or industry-specific skills, and these skills are permanently learned, perhaps with a small decay rate, if used in the same occupation. A change of occupation or industry where such skills are unused makes them less valuable and, possibly, makes them depreciate much faster. In job ladder models, earnings risk is asymmetric, job-loss risk affecting workers at the top of the ladder while workers at the bottom face opportunities to move up (Lise (2013)). In a recent model of earnings losses upon displacement, Huckfeldt (2016) included an unskilled sector that tends to absorb laid-off workers. With low probability, workers have the opportunity to escape this sector and move to the skilled sector that features a job ladder. The escape from the unskilled sector is the event that wipes out the memory of the past bad shocks. From an empirical perspective, "unusual" shocks could correspond to job losses, changes of career, or health shocks. If such life-changing events are occasionally experienced by households, one would expect their predictive probability distributions over future income to feature nonlinear dynamic asymmetries.

Consider large, negative "unusual" income shocks, which not only have a direct effect but also cancel out the persistence of a good income history. For example, a worker hit by an adverse occupation-specific shock might find her skills less valuable. In that case, her previous earnings history may matter much less after the shock. Using a parallel with the macroeconomic literature on disaster risk, these shocks could be called "microeconomic disasters." While macroeconomic disasters could have potentially large effects on saving behavior (Rietz (1988), Barro (2006)), they are so unlikely that they are statistically elusive events. In contrast, disasters at the micro level happen all the time to some individuals and therefore their dynamic consequences may have clear-cut empirical content. ${ }^{6}$

Such features are prominent in the empirical results that we report in this paper, and they are all at odds with linear models commonly used in the earnings dynamics literature. Moreover, despite recent advances on models of distributional earnings dynamics (e.g., Meghir and Pistaferri (2004) or Botosaru and Sasaki (2015)), existing models do not seem well-suited to capture the nonlinear transmission of income shocks that we uncover.

Our second contribution is to develop an estimation framework to assess how consumption responds to earnings shocks in the data. In the baseline analysis, we model the consumption policy rule as an age-dependent nonlinear function of assets, unobserved tastes, and the persistent and transitory earnings components. We motivate our specification using a standard life-cycle model of consumption and saving with incomplete markets (as in Huggett (1993), e.g.). In this model, as we illustrate through a small simulation exercise, a nonlinear earnings process with dynamic skewness of the type we uncover in our conditional quantile analysis will have qualitatively different implications for the level and distribution of consumption and assets over the life-cycle in comparison to a linear earnings model.

The empirical consumption rule we develop is nonlinear, thus allowing for age-specific interactions between asset holdings and the earnings components. However, unlike fully specified structural approaches, we model the consumption rule nonparametrically, leaving functional forms unrestricted. This modeling approach allows capturing an array of response coefficients that provides a rich picture of the extent of consumption insurance in the data. Moreover, there is no need for approximation arguments as we directly estimate

\footnotetext{
${ }^{6}$ The notion of "micro disasters" is also related to Castañeda, Díaz-Giménez, and Ríos-Rull (2003), who argued that allowing for a substantial probability of downward risk for high-income households may help explain wealth inequality. See also Constandinides and Gosh (2017) and Schmidt (2015), who emphasized the asset pricing implications of income risk asymmetries.
} 
the nonlinear consumption rule. Our consumption rule allows for unobserved household heterogeneity. We also show how to extend the framework to allow for advance information on earnings shocks and habits in consumption.

A virtue of our consumption framework is its ability to produce new empirical quantities, such as nonparametric marginal propensities to consume, that narrow the gap between policy-relevant evidence and structural modeling. At the same time, in the absence of further assumptions, the model can be thought of as semi-structural and cannot generally be used to perform policy counterfactuals. As an example, in order to assess the impact of a change in the earnings process on consumption dynamics, one would need to take a precise stand on preferences and expectations, among other factors. Although, given our goal to document nonlinear effects, we do not impose such assumptions in this paper, economic structure and/or measured expectations could be added to our framework in order to conduct policy evaluation exercises.

Beyond the traditional covariance methods that have dominated the literature, new econometric techniques are needed to study our nonlinear model of earnings and consumption. Nonparametric identification can be established in our setup by building on a recent literature on nonlinear models with latent variables. Identification of the earnings process follows $\mathrm{Hu}$ and Schennach (2008) and Wilhelm (2015). ${ }^{7}$ Identification of the consumption rule relies on novel arguments, which extend standard instrumental-variables methods (as in Blundell, Pistaferri, and Preston (2008), e.g.) to our nonparametric setup.

To devise a tractable estimation strategy, we rely on the approach introduced by Arellano and Bonhomme (2016), adapted to a setup with time-varying latent variables. Wei and Carroll (2009) introduced a related estimation strategy in a cross-sectional context. The approach combines quantile regression methods, which are well-suited to capture nonlinear effects of earnings shocks, with regression methods on bases of functions, which are well-suited to flexibly model conditional distributions. ${ }^{8}$ To deal with the presence of the latent earnings components, we use a sequential estimation algorithm that consists in iterating between quantile regression estimation, and draws from the posterior distribution of the latent persistent components of earnings.

We take the earnings and consumption model to data from the Panel Study of Income Dynamics (PSID) for 1999-2009 and focus on working-age families. Unlike earlier waves of the PSID, these data contain enhanced information on asset holdings and consumption expenditures in addition to labor earnings; see Blundell, Pistaferri, and Saporta-Eksten (2016), for example. This is the first household panel to include detailed information on consumption and assets across the life-cycle for a representative sample of households. Although we abstract from labor supply, our modeling and estimation approach makes full use of the availability of panel information on earnings, consumption, and assets. In addition, the quantile regression specifications that we use allow us to obtain rather precise estimates, despite the flexibility of the model and the moderate sample size.

Our empirical results show that the impact of earnings shocks varies substantially across households' earnings histories, and that this nonlinearity is a driver of heterogeneous consumption responses. Earnings data show the presence of nonlinear persistence, where "unusual" positive shocks for low earnings households, and negative shocks for high earnings households, are associated with lower persistence than other shocks. That is, such shocks have a higher propensity to wipe out the impact of the previous earnings history.

\footnotetext{
${ }^{7}$ Lochner and Shin (2014) relied on related techniques to establish identification of a different nonlinear model of earnings.

${ }^{8}$ Misra and Surico (2014) used quantile methods to document the consumption responses associated with tax rebates.
} 
Related to this, we find that conditional log-earnings distributions are asymmetric, skewed to the right (respectively, left) for households at the bottom (resp., top) of the income distribution. Although most of our results are based on PSID data, we show that similar empirical patterns hold in Norwegian administrative earnings data.

Regarding consumption, we find a significant degree of insurability of shocks to the persistent earnings components. We also uncover asymmetries in consumption responses to earnings shocks that hit households at different points of the income distribution. Last, we find that assets play a role in the insurability of earnings shocks.

The literature on earnings dynamics is vast. Recent work has focused on nonGaussianity (Geweke and Keane (2000), Bonhomme and Robin (2010)) and heterogeneity (Browning, Ejrnaes, and Alvarez (2010)). The nonlinear earnings persistence that we uncover is consistent with findings on U.S. administrative tax records, such as Guvenen, Ozcan, and Song (2014) and especially recent independent work by Guvenen, Karahan, Ozcan, and Song (2015). Relative to this growing body of research, the fact that our quantile-based methods are able to uncover previously unknown results in PSID survey data, and that these results also hold in administrative "big data" sets, is important because PSID uniquely provides joint longitudinal data on wealth, income, and expenditures at household level. This allows us to conduct a joint empirical analysis of earnings and consumption patterns. ${ }^{9}$

The outline of the paper is as follows: In the next section, we describe the earnings process and develop our measure of nonlinear persistence. Section 3 lays out the consumption model and defines a general representation of partial insurance to earnings shocks. In Section 4, we establish identification of the model. Section 5 describes our estimation strategy and the panel data set. In Section 6, we present our empirical results. Section 7 concludes with a summary and some directions for future research. The Supplemental Material (Arellano, Blundell, and Bonhomme (2017)) contains additional results.

\section{MODEL (I): EARNINGS PROCESS}

We start by describing our nonlinear model of earnings dynamics. In the next section, we will present the consumption model.

\subsection{The Model}

We consider a cohort of households, $i=1, \ldots, N$, and denote as $t$ the age of the household head (relative to $t=1$ ). Let $Y_{i t}$ be the pre-tax labor earnings of household $i$ at age $t$, and let $y_{i t}$ denote $\log -Y_{i t}$, net of a full set of age dummies. We decompose $y_{i t}$ as follows: ${ }^{10}$

$$
y_{i t}=\eta_{i t}+\varepsilon_{i t}, \quad i=1, \ldots, N, t=1, \ldots, T,
$$

where the probability distributions of $\eta$ 's and $\varepsilon$ 's are absolutely continuous.

The first, persistent component $\eta_{i t}$ is assumed to follow a general first-order Markov process. We denote the $\tau$ th conditional quantile of $\eta_{i t}$ given $\eta_{i, t-1}$ as $Q_{t}\left(\eta_{i, t-1}, \tau\right)$, for

\footnotetext{
${ }^{9}$ Our nonlinear earnings model featuring asymmetric persistence is also related to time-series regimeswitching models that are popular to analyze business-cycle dynamics; see, for example, Evans and Wachtel's (1993) model of inflation uncertainty and Teräsvirta (1994) on smooth transition autoregressive models.

${ }^{10}$ Model (1) is additive in $\eta$ and $\varepsilon$. Given our nonlinear approach, it is in principle possible to allow for interactions between the two earnings components, for example in $y_{i t}=H_{t}\left(\eta_{i t}, \varepsilon_{i t}\right)$, where identification could be established along the lines of $\mathrm{Hu}$ and Shum (2012).
} 
each $\tau \in(0,1)$. The following representation is then without loss of generality:

$$
\eta_{i t}=Q_{t}\left(\eta_{i, t-1}, u_{i t}\right), \quad\left(u_{i t} \mid \eta_{i, t-1}, \eta_{i, t-2}, \ldots\right) \sim \operatorname{Uniform}(0,1), \quad t=2, \ldots, T .
$$

The dependence structure of the $\eta$ process is not restricted beyond the first-order Markov assumption. The identification assumptions will only require $\eta$ 's to be dependent over time, without specifying a particular (parametric) form of dependence.

The second, transitory component $\varepsilon_{i t}$ is assumed to have zero mean, to be independent over time and independent of $\eta_{i s}$ for all $s$. Even though more general moving average representations are commonly used in the literature, the biennial nature of the PSID data makes this assumption more plausible. Model (1)-(2) is intended as a representation of the uncertainty about persistent and transitory labor income in future periods that households face when deciding how much to spend and save. Our approach can be extended to allow for a higher-order Markov $\eta$ process or a moving average $\varepsilon$ component, provided additional time periods are available, and for an unobserved time-invariant householdspecific effect in addition to the two latent time-varying components $\eta$ and $\varepsilon$ (as done in the Supplemental Material).

Survey data like the PSID are often contaminated with errors (Bound, Brown, and Mathiowetz (2001)). In the absence of additional information, it is not possible to disentangle the transitory innovation from classical measurement error. Thus, an interpretation of our estimated distribution of $\varepsilon_{i t}$ is that it represents a mixture of transitory shocks and measurement error. ${ }^{11}$

Both earnings components are assumed mean independent of age $t$. However, the conditional quantile functions $Q_{t}$, and the marginal distributions of $\varepsilon_{i t}$, may all depend on $t$. For a given cohort of households, age and calendar time are perfectly collinear, so this dependence may capture age effects as well as aggregate shocks. The distribution of the initial condition $\eta_{i 1}$ is left unrestricted.

An important special case of model (1)-(2) is obtained when

$$
y_{i t}=\eta_{i t}+\varepsilon_{i t}, \quad \eta_{i t}=\eta_{i, t-1}+v_{i t},
$$

that is, when $\eta_{i t}$ follows a random walk. When $v_{i t}$ is independent of $\eta_{i, t-1}$ and has cumulative distribution function $F_{t}$, (2) becomes: $\eta_{i t}=\eta_{i, t-1}+F_{t}^{-1}\left(u_{i t}\right)$. We will refer to the random walk plus independent shock as the canonical model of earnings dynamics.

\subsection{Nonlinear Dynamics}

Model (1)-(2) allows for nonlinear dynamics of earnings. Here we focus on the ability of this specification to capture nonlinear persistence, and general forms of conditional heteroscedasticity.

Nonlinear Persistence. We introduce the following quantities:

$$
\rho_{t}\left(\eta_{i, t-1}, \tau\right)=\frac{\partial Q_{t}\left(\eta_{i, t-1}, \tau\right)}{\partial \eta}, \quad \rho_{t}(\tau)=\mathbb{E}\left[\frac{\partial Q_{t}\left(\eta_{i, t-1}, \tau\right)}{\partial \eta}\right],
$$

where $\partial Q_{t} / \partial \eta$ denotes the partial derivative of $Q_{t}$ with respect to its first argument and the expectation is taken with respect to the distribution of $\eta_{i, t-1}$.

\footnotetext{
${ }^{11}$ If additional information were available and the marginal distribution of a classical measurement error were known, one could recover the distribution of $\varepsilon_{i t}$ using a deconvolution argument. The estimation algorithm can be modified to deal with this case.
} 
The $\rho$ 's in (4) are measures of nonlinear persistence of the $\eta$ component. ${ }^{12} \rho_{t}\left(\eta_{i, t-1}, \tau\right)$ measures the persistence of $\eta_{i, t-1}$ when it is hit by a current shock $u_{i t}$ that has rank $\tau$. This quantity depends on the lagged component $\eta_{i, t-1}$, and on the percentile of the shock $\tau$. Average persistence across $\eta$ values is $\rho_{t}(\tau)$. Note that, while the shocks $u_{i t}$ are i.i.d. by construction, they may greatly differ in the earnings persistence associated with them. The $\rho$ 's are thus measures of persistence of earnings histories.

In the canonical model of earnings dynamics (3) where $\eta_{i t}$ is a random walk, $\rho_{t}\left(\eta_{i, t-1}, \tau\right)=1$ irrespective of $\eta_{i, t-1}$ and $\tau$. In contrast, in model (2), the persistence of $\eta_{i, t-1}$ may depend on the magnitude and direction of the shock $u_{i t}$. As a result, the persistence of a shock to $\eta_{i, t-1}$ depends on the size and sign of current and future shocks $u_{i t}, u_{i, t+1}, \ldots$ In particular, our model allows particular shocks to wipe out the memory of past shocks. As reviewed in the Introduction, labor market models of the job ladder and occupational mobility can involve workers facing an increasing risk of a large fall in earnings, while those recently laid off have a small probability of a positive shock that takes them into a skilled job where they can advance along the ladder. The interaction between the shock $u_{i t}$ and the lagged persistent component $\eta_{i, t-1}$ is a central feature of our nonlinear approach and, as we show below, it has substantive implications for consumption decisions.

It is useful to consider the following specification of the quantile function:

$$
Q_{t}\left(\eta_{i, t-1}, \tau\right)=\alpha_{t}(\tau)+\beta_{t}(\tau)^{\prime} h\left(\eta_{i, t-1}\right)
$$

where $h$ is a multi-valued function. Our empirical specification will be based on (5), taking the components of $h$ in a polynomial basis of functions. Persistence and average persistence in (5) are, respectively,

$$
\rho_{t}\left(\eta_{i, t-1}, \tau\right)=\beta_{t}(\tau)^{\prime} \frac{\partial h\left(\eta_{i, t-1}\right)}{\partial \eta}, \quad \rho_{t}(\tau)=\beta_{t}(\tau)^{\prime} \mathbb{E}\left[\frac{\partial h\left(\eta_{i, t-1}\right)}{\partial \eta}\right],
$$

thus allowing shocks to affect the persistence of $\eta_{i, t-1}$ in a flexible way. This measure is related to quantile autoregression parameters, as in Koenker and Xiao (2006).

Conditional Heteroscedasticity. As model (2) does not restrict the form of the conditional distribution of $\eta_{i t}$ given $\eta_{i, t-1}$, it allows for general forms of heteroscedasticity. In particular, a measure of period- $t$ uncertainty generated by the presence of shocks to the persistent earnings component is, for some $\tau \in(1 / 2,1), \sigma_{t}\left(\eta_{i, t-1}, \tau\right)=Q_{t}\left(\eta_{i, t-1}, \tau\right)-$ $Q_{t}\left(\eta_{i, t-1}, 1-\tau\right)$. For example, in the canonical model (3) with $v_{i t} \sim \mathcal{N}\left(0, \sigma_{v_{t}}^{2}\right)$, we have $\sigma_{t}\left(\eta_{i, t-1}, \tau\right)=2 \sigma_{v_{t}} \Phi^{-1}(\tau) .^{13}$

In addition, the model allows for conditional skewness and kurtosis in $\eta_{i t}$. Along the lines of the skewness measure proposed by Kim and White (2004), one can consider, for

\footnotetext{
${ }^{12}$ Note that $\rho_{t}\left(\eta_{i, t-1}, \tau\right)$ may be positive or negative, and may exceed 1 in absolute value. As a simple illustration, if $\ln \left(\eta_{i t}\right)$ is a random walk with standard Gaussian innovations, $\eta_{i t}$ itself is a multiplicative random walk, for which derivative measures of persistence in (4) do not vary with lagged $\eta$ but vary with the value of the shock. For example, at the median shock the derivative is 1 , but at the bottom quartile shock the derivative is around 0.5 , and at the top quartile it is around 2 .

${ }^{13}$ The shock $u_{i t}$ is a rank. A persistent shock of a magnitude comparable to $\eta_{i t}$ can be constructed, among other ways, as $\zeta_{i t}=Q_{t}\left(m_{t}, u_{i t}\right)$ where $m_{t}$ is the median of $\eta_{i t}$.
} 
(a) PSID data

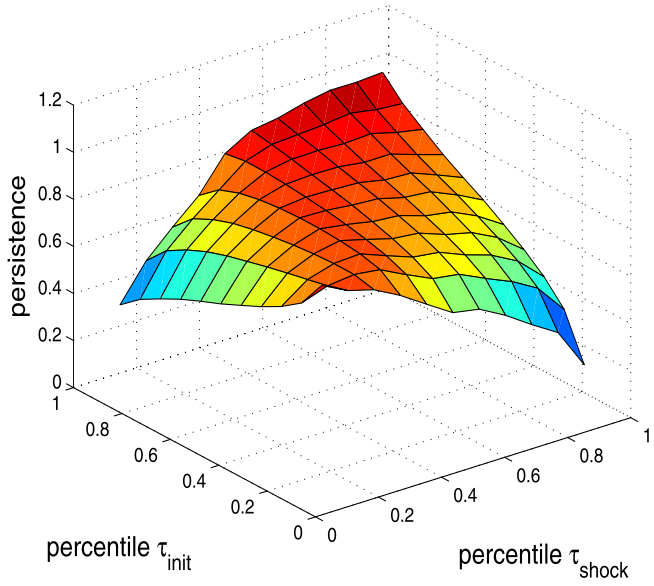

(b) Norwegian administrative data

FIGURE 1.-Quantile autoregressions of log-earnings. Note: Residuals $y_{i t}$ of log pre-tax household labor earnings, Age 25-60 1999-2009 (U.S.), Age 25-60 2005-2006 (Norway). See Section 6 and Appendix C for the list of controls. Estimates of the average derivative of the conditional quantile function of $y_{i t}$ given $y_{i, t-1}$ with respect to $y_{i, t-1}$. Quantile functions are specified as third-order Hermite polynomials. Source: The Norwegian results are part of the project on 'Labour Income Dynamics and the Insurance from Taxes, Transfers and the Family'. See Appendix C.

some $\tau \in(1 / 2,1),{ }^{14}$

$$
s k_{t}\left(\eta_{i, t-1}, \tau\right)=\frac{Q_{t}\left(\eta_{i, t-1}, \tau\right)+Q_{t}\left(\eta_{i, t-1}, 1-\tau\right)-2 Q_{t}\left(\eta_{i, t-1}, \frac{1}{2}\right)}{Q_{t}\left(\eta_{i, t-1}, \tau\right)-Q_{t}\left(\eta_{i, t-1}, 1-\tau\right)} .
$$

The empirical estimates below suggest that conditional skewness is a feature of the earnings process.

Preliminary Evidence on Nonlinear Persistence. Suggestive evidence of nonlinearity in the persistence of earnings can be seen from Figure 1. This figure plots estimates of the average derivative, with respect to last period income $y_{i, t-1}$, of the conditional quantile function of current income $y_{i t}$ given $y_{i, t-1}$. This average derivative effect is a measure of persistence analogous to $\rho_{t}$ in (4), except that here we use residuals $y_{i t}$ of log pre-tax household labor earnings on a set of demographics (including education and a polynomial in the age of the household head) as outcome variables. Given the nature of the PSID sample, panel (a) features biennial persistence estimates. On the two horizontal axes, we report the percentile of $y_{i, t-1}$ (" $\tau_{\text {init }}$ "), and the percentile of the innovation of the quantile process (" $\tau_{\text {shock }}$ "). For estimation, we use a series quantile specification, as in $(5)$, based on a third-order Hermite polynomial.

This simple descriptive analysis not only shows the similarity in the patterns of the nonlinearity of household earnings in both the PSID household panel survey data and

${ }^{14}$ Similarly, a measure of conditional kurtosis is, for some $\alpha<1-\tau$,

$$
k u r_{t}\left(\eta_{i, t-1}, \tau, \alpha\right)=\frac{Q_{t}\left(\eta_{i, t-1}, 1-\alpha\right)-Q_{t}\left(\eta_{i, t-1}, \alpha\right)}{Q_{t}\left(\eta_{i, t-1}, \tau\right)-Q_{t}\left(\eta_{i, t-1}, 1-\tau\right)} .
$$


in the annual population register data from Norway; it also suggests differences in the impact of an innovation to the quantile process $\left(\tau_{\text {shock }}\right)$ according to both the direction and magnitude of $\tau_{\text {shock }}$ and the percentile of the past level of income $\tau_{\text {init }}$. Persistence of earnings history is highest when high-earnings households (i.e., high $\tau_{\text {init }}$ ) are hit by a good shock (high $\tau_{\text {shock}}$ ), and when low-earnings households (i.e., low $\tau_{\text {init }}$ ) are hit by a bad shock (low $\tau_{\text {shock }}$ ). In both cases, estimated persistence is close to $0.9-1$. In contrast, bad shocks hitting high-earnings households, and good shocks hitting low-earnings ones, are associated with much lower persistence of earnings history, as low as 0.3-0.4. In Section 6, we will see that our nonlinear model that separates transitory shocks from the persistent component reproduces the nonlinear persistence patterns of Figure 1 for the PSID panel survey data and the Norwegian population register data.

\section{MODEL (II): CONSUMPTION RULE}

In order to motivate our empirical specification of the consumption function, we start by describing a standard stochastic life-cycle consumption model. We then use this setup to derive the form of the policy rule for household consumption, and describe the empirical consumption model that we will take to the data.

\subsection{The Consumption Rule in a Simple Life-Cycle Model}

We consider a theoretical framework where households act as single agents. Throughout their lifetime, households have access to a single risk-free, one-period bond whose constant return is $1+r$, and, at age $t$, face a period-to-period budget constraint

$$
A_{i t}=(1+r) A_{i, t-1}+Y_{i, t-1}-C_{i, t-1},
$$

where $A_{i t}, Y_{i t}$, and $C_{i t}$ denote assets, income, and consumption, respectively. Family logearnings are given by $\ln Y_{i t}=\kappa_{t}+\eta_{i t}+\varepsilon_{i t}$, where $\kappa_{t}$ is a deterministic age profile, and $\eta_{i t}$ and $\varepsilon_{i t}$ are persistent and transitory earnings components, respectively. At age $t$, agents know $\eta_{i t}, \varepsilon_{i t}$ and their past values, but not $\eta_{i, t+1}$ or $\varepsilon_{i, t+1}$, so there is no advance information. All distributions are known to households, and there is no aggregate uncertainty. In each period $t$ in the life-cycle, the optimization problem is represented by the Bellman equation

$$
V_{t}\left(A_{i t}, \eta_{i t}, \varepsilon_{i t}\right)=\max _{C_{i t}} u\left(C_{i t}\right)+\beta \mathbb{E}_{t}\left[V_{t+1}\left(A_{i, t+1}, \eta_{i, t+1}, \varepsilon_{i, t+1}\right)\right],
$$

where $u(\cdot)$ is agents' utility, and $\beta$ is the discount factor. An important element in (8) is the conditional distribution of the Markov component $\eta_{i, t+1}$ given $\eta_{i t}$, which enters the expectation.

In a nonlinear earnings model such as (1)-(2), the presence of "unusual" shocks to earnings may lead to precautionary motives that induce high-income households to save more than they would do under a linear earnings model. Even with certainty equivalent preferences, under model (1)-(2) the discounting applied to persistent shocks will be state-dependent. In Section S1 of the Supplemental Material, we illustrate these theoretical mechanisms in a two-period version of the model. In Section S2, we report the results of a simulation exercise based on the life-cycle model. We compare the canonical linear earnings model with an earnings model that features the presence of positive and negative "unusual" shocks. In the simulated economy, we find that an implication of the nonlinear 
earnings process is to reduce consumption among households on higher incomes. In particular, a negative shock for those on higher incomes reduces the persistence of the past and consequently is more damaging in terms of expected future incomes, thus inducing higher saving, higher wealth accumulation, and lower consumption. ${ }^{15}$

In such a life-cycle model with uncertainty, the consumption rule will have the form

$$
C_{i t}=G_{t}\left(A_{i t}, \eta_{i t}, \varepsilon_{i t}\right)
$$

for some age-dependent function $G_{t}$. We will base our empirical specification on (9). The consumption rule at age $t$ will be of this nonparametric form where the state variables at $t$ are period- $t$ assets and the latent earnings components. ${ }^{16}$

In documenting dynamic patterns of consumption and earnings, one strategy is to take a stand on the functional form of the utility function and the distributions of the shocks, and to calibrate or estimate the model's parameters by comparing the model's predictions with the data. Another strategy is to linearize the Euler equation, with the help of the budget constraint; with a linear approximated problem at hand, standard covariancebased methods may be used for estimation. Our approach differs from those strategies as we directly estimate the nonlinear consumption rule (9). Doing so, we avoid linearized first-order conditions, and we estimate a flexible rule that is consistent with the above life-cycle consumption model. This approach allows documenting a rich set of derivative effects, thus shedding light on the patterns of consumption responses in the data.

\subsection{Empirical Consumption Rule}

Consider a cohort of households. Let $c_{i t}$ denote log-consumption net of age dummies. Similarly, let $a_{i t}$ denote log-assets net of age dummies. Our empirical specifications are based on

$$
c_{i t}=g_{t}\left(a_{i t}, \eta_{i t}, \varepsilon_{i t}, \nu_{i t}\right), \quad t=1, \ldots, T .
$$

The $\nu_{i t}$ are unobserved arguments of the consumption function, in addition to assets and the latent earnings components. In the specification without unobserved individual heterogeneity, $\nu_{i t}$ are independent across periods and independent of $\left(a_{i t}, \eta_{i t}, \varepsilon_{i t}\right)$, and $g_{t}$ is monotone in $\nu$. An economic interpretation for $\nu$ is as a taste shifter that increases marginal utility. In the single-asset life-cycle model of Section 3.1, monotonicity is implied by the Bellman equation, provided $\frac{\partial u\left(C, \nu^{\prime}\right)}{\partial C}>\frac{\partial u(C, \nu)}{\partial C}$ for all $C$ if $\nu^{\prime}>\nu$, where the household's utility function is $u(C, \nu)$. Without loss of generality, we normalize the marginal distribution of $\nu_{i t}$ to be standard uniform in each period. From an empirical perspective, the presence of the taste shifters $\nu_{i t}$ in the consumption rule (10) may also partly capture measurement error in consumption expenditures. In the specification with unobserved individual heterogeneity, $\nu_{i t}$ comprises two components: a time-invariant latent household factor, and an i.i.d. uniform component independent of the latter. Consumption is monotone in the second component, while being fully nonlinear in the first.

\footnotetext{
${ }^{15}$ In addition, in Section S2 of the Supplemental Material, we report simulation results based on the same life-cycle model, using as input the earnings process we estimate on the PSID.

${ }^{16}$ Our approach may be extended to allow for habits or advance information, through simple modifications of the vector of state variables. There could also be additional borrowing constraints in each period. In that case, the nonparametric consumption rule in (9) would no longer be differentiable. The derivative effects defined below require differentiability of $g_{t}$.
} 
Clearly, the net assets variable $a_{i t}$ is not exogenous. In order to ensure identification, it suffices to specify $a_{i t}$ as sequentially exogenous (or "predetermined"). We will specify assets as a function of lagged assets, consumption, earnings, the persistent earnings component $\eta$, and age, as follows:

$$
a_{i t}=h_{t}\left(a_{i, t-1}, c_{i, t-1}, y_{i, t-1}, \eta_{i, t-1}, v_{i t}\right),
$$

where $h_{t}$ is an age-specific function which is increasing in its last argument, and $v_{i t}$ are i.i.d. uniform independent of the other arguments. Note that the standard linear asset rule (7) is a special case of (11), so imposing (7) is not needed for identification or estimation. Taking a stand on the budget constraint will, however, be required in order to simulate the impact of earnings shocks over the life-cycle.

Derivative Effects. Average consumption, for given values of asset holdings and earnings components, is

$$
\mathbb{E}\left[c_{i t} \mid a_{i t}=a, \eta_{i t}=\eta, \varepsilon_{i t}=\varepsilon\right]=\mathbb{E}\left[g_{t}\left(a, \eta, \varepsilon, \nu_{i t}\right)\right] .
$$

Our framework allows us to document how average consumption varies as a function of assets and the two earnings components, and over the life-cycle. In particular, the average derivative of consumption with respect to $\eta$ is

$$
\phi_{t}(a, \eta, \varepsilon)=\mathbb{E}\left[\frac{\partial g_{t}\left(a, \eta, \varepsilon, \nu_{i t}\right)}{\partial \eta}\right] .
$$

The parameter $\phi_{t}(a, \eta, \varepsilon)$ reflects the degree of insurability of shocks to the persistent earnings component. ${ }^{17}$ We will document how this new measure of partial insurance varies over the life-cycle, and how it depends on households' asset holdings, by reporting estimates of the average derivative effect $\bar{\phi}_{t}(a)=\mathbb{E}\left[\phi_{t}\left(a, \eta_{i t}, \varepsilon_{i t}\right)\right]$. The quantity $1-\bar{\phi}_{t}(a)$ is then a general measure of the degree of consumption insurability of shocks to the persistent earnings component, as a function of age and assets.

Dynamic Effects of Earnings Shocks on Consumption. Other measures of interest are the effects of an earnings shock $u_{i t}$ to the $\eta$ component on the consumption profile $c_{i, t+s}$, $s \geq 0$. For example, the contemporaneous effect can be computed, using the chain rule and equation (12), as

$$
\mathbb{E}\left[\left.\frac{\partial}{\partial u}\right|_{u=\tau} g_{t}\left(a, Q_{t}(\eta, u), \varepsilon, \nu_{i t}\right)\right]=\phi_{t}\left(a, Q_{t}(\eta, \tau), \varepsilon\right) \frac{\partial Q_{t}(\eta, \tau)}{\partial u} .
$$

This derivative effect depends on $\eta$ through the insurance coefficient $\phi_{t}$, but also through the quantity $\frac{\partial Q_{t}(\eta, \tau)}{\partial u}$ as the earnings model allows for general forms of conditional heteroscedasticity and skewness. The quantity $\frac{\partial Q_{t}(\eta, \tau)}{\partial u}$ measures the responsiveness of earnings to a shock $u_{i t}$ on impact. Note that its derivative with respect to $\eta$ is equal to $\frac{\partial \rho_{t}(\eta, \tau)}{\partial \tau}$, where $\rho_{t}(\eta, \tau)$ is our persistence measure. In the empirical analysis, we will report finitedifference counterparts to these derivative effects ("impulse responses"), and find an asymmetric impact of earnings shocks at different points of the income distribution.

Multiple Assets. Our approach can be generalized to represent the consumption policy function of a model with multiple assets differing in the stochastic properties of their returns. An example is a model that distinguishes between a risky asset and a risk-free

\footnotetext{
${ }^{17}$ Likewise, the average derivative with respect to $\varepsilon$ is $\psi_{t}(a, \eta, \varepsilon)=\mathbb{E}\left[\frac{\partial g_{t}\left(a, \eta, \varepsilon, \nu_{i t}\right)}{\partial \varepsilon}\right]$.
} 
asset, as often used in studies of household portfolios (e.g., Alan (2012)). A version of the consumption rule (10) with two assets would be consistent with this type of model as long as excess returns of the risky asset are not heterogeneous across households. In the presence of kinks induced by participation or transaction costs, our empirical consumption rule would capture a smoothed approximation.

The consumption rule (10) can also be extended to the Kaplan and Violante (2014) model of wealthy "hand-to-mouth" consumers. In that framework, the consumption policy rule is a nonlinear function of assets disaggregated into liquid and illiquid parts. Access to the illiquid, higher return asset involves a transaction cost. The separate assets interact in nonlinear ways with earnings. In this dynamic choice environment, the nonlinearity in the consumption model (10) incorporating the two separate assets can provide a smoothed approximation to the complex paths involved in the Kaplan and Violante (2014) composite consumption function. ${ }^{18}$

\section{IDENTIFICATION}

The earnings and consumption models take the form of nonlinear state-space models. A series of recent papers (notably Hu and Schennach (2008), and Hu and Shum (2012)) has established conditions under which nonlinear models with latent variables are nonparametrically identified under conditional independence restrictions. Techniques developed in this literature can be used in order to establish identification of the models we consider.

\subsection{Earnings Process}

Consider model (1)-(2), where $\eta_{i t}$ is a Markovian persistent component and $\varepsilon_{i t}$ are independent over time and independent of the $\eta$ 's. We assume that the data contain $T$ consecutive periods, $t=1, \ldots, T$. So, for a given cohort of households, $t=1$ corresponds to the age at which the household head enters the sample, and $t=T$ corresponds to the last period of observation. ${ }^{19}$ For that cohort, our aim is to identify the joint distributions of $\left(\eta_{i 1}, \ldots, \eta_{i T}\right)$ and $\left(\varepsilon_{i 1}, \ldots, \varepsilon_{i T}\right)$ given i.i.d. data from $\left(y_{i 1}, \ldots, y_{i T}\right)$. Four periods are needed in order to identify at least one Markov transition $Q_{t}$.

Conditions for the nonparametric identification of the earnings process are direct consequences of the analysis in Hu and Schennach (2008) and Wilhelm (2015). We provide such conditions in Appendix A. Identification is derived under several high-level assumptions. In particular, the distributions of $\left(y_{i t} \mid y_{i, t-1}\right)$ and $\left(\eta_{i t} \mid y_{i, t-1}\right)$ both need to satisfy completeness conditions. For example, the first condition requires that the only function $h$ (in a suitable functional space) satisfying $\mathbb{E}\left[h\left(y_{i t}\right) \mid y_{i, t-1}\right]=0$ be $h=0$. This requires that $\eta_{i, t-1}$ and $\eta_{i t}$ be statistically dependent, albeit without specifying the form of that dependence. An intuition for this is that if $\eta$ 's were independent over time, there would be no way to distinguish them from the transitory $\varepsilon$ 's. Completeness is commonly assumed in nonparametric instrumental variables problems (Newey and Powell (2003)).

\footnotetext{
${ }^{18} \mathrm{~A}$ separate question of interest, but one that is beyond the scope of this paper, concerns the identification of the latent consumption policy functions associated with the agent's accessing or not accessing the illiquid asset. Such a question could be posed if we observed a time-varying indicator of whether or not consumers access their illiquid assets.

${ }^{19}$ We consider a balanced panel for simplicity, but our arguments can be extended to unbalanced panels.
} 


\subsection{Consumption Rule}

Let us now turn to the identification of the consumption rule (10), starting with the case without unobserved heterogeneity. We make the following assumptions, where we denote $z_{i}^{t}=\left(z_{i 1}, \ldots, z_{i t}\right)$.

AssumPTION 1: For all $t \geq 1$,

(i) $u_{i, t+s}$ and $\varepsilon_{i, t+s}$, for all $s \geq 0$, are independent of $a_{i}^{t}, \eta_{i}^{t-1}$, and $y_{i}^{t-1} . \varepsilon_{i 1}$ is independent of $a_{i 1}$ and $\eta_{i 1}$.

(ii) $a_{i, t+1}$ is independent of $\left(a_{i}^{t-1}, c_{i}^{t-1}, y_{i}^{t-1}, \eta_{i}^{t-1}\right)$ conditional on $\left(a_{i t}, c_{i t}, y_{i t}, \eta_{i t}\right)$.

(iii) The taste shifter $\nu_{i t}$ in (10) is independent of $\eta_{i 1},\left(u_{i s}, \varepsilon_{i s}\right)$ for all $s, \nu_{i s}$ for all $s \neq t$, and $a_{i}^{t}$.

Part (i) in Assumption 1 requires current and future earnings shocks, which are independent of past components of earnings, to be independent of current and past asset holdings as well. At the same time, we let $\eta_{i 1}$ and $a_{i 1}$ be arbitrarily dependent. This is important, because asset accumulation upon entry in the sample may be correlated with past earnings shocks. Part (ii) is a first-order Markov condition on asset accumulation. It is satisfied in a standard life-cycle model with one single risk-less asset; see equation (7). The assumption also holds in such a model when the interest rate $r_{t}$ is time-varying and known to households. More generally, the assumption allows the latent components of earnings $\eta_{i t}$ and $\varepsilon_{i t}$ to affect asset holdings separately, as in (11). Last, part (iii) requires taste shifters to be independent over time, independent of earnings components, and independent of current and past assets. In particular, this rules out the presence of unobserved heterogeneity in consumption. We will relax this condition in the next subsection.

The identification argument proceeds in a sequential way. Starting with the first period, letting $y_{i}=\left(y_{i 1}, \ldots, y_{i T}\right)$, and using $f$ as a generic notation for a density function, we have

$$
f\left(a_{1} \mid y\right)=\int f\left(a_{1} \mid \eta_{1}\right) f\left(\eta_{1} \mid y\right) d \eta_{1},
$$

where we have used that, by Assumption 1(i), $f\left(a_{1} \mid \eta_{1}, y\right)$ and $f\left(a_{1} \mid \eta_{1}\right)$ coincide. We can rewrite (13) as

$$
f\left(a_{1} \mid y\right)=\mathbb{E}\left[f\left(a_{1} \mid \eta_{i 1}\right) \mid y_{i}=y\right],
$$

where the expectation is taken with respect to the density of $\eta_{i 1}$ given $y_{i}$, for a fixed value $a_{1}$. Hence, provided the distribution of $\left(\eta_{i 1} \mid y_{i}\right)$ (which is identified from the earnings process; see above) is complete, the density $f\left(a_{1} \mid \eta_{1}\right)$ is identified from (14).$^{20}$ Note that the density $f\left(a_{1}, \eta_{1} \mid y\right)=f\left(a_{1} \mid \eta_{1}\right) f\left(\eta_{1} \mid y\right)$ is also identified.

We then have, using the consumption rule and Assumption 1(iii),

$$
f\left(c_{1} \mid a_{1}, y\right)=\int f\left(c_{1} \mid a_{1}, \eta_{1}, y_{1}\right) f\left(\eta_{1} \mid a_{1}, y\right) d \eta_{1},
$$

or equivalently,

$$
f\left(c_{1} \mid a_{1}, y\right)=\mathbb{E}\left[f\left(c_{1} \mid a_{i 1}, \eta_{i 1}, y_{i 1}\right) \mid a_{i 1}=a_{1}, y_{i}=y\right],
$$

\footnotetext{
${ }^{20} \mathrm{In}$ fact, given that we are working with bounded density functions, it is sufficient that the distribution of $\left(\eta_{i 1} \mid y_{i}\right)$ be boundedly complete; see Blundell, Chen, and Kristensen (2007) for analysis and discussion.
} 
where the conditional expectation is taken at fixed $c_{1}$. Under completeness in $\left(y_{i 2}, \ldots, y_{i T}\right)$ of the distribution of $\left(\eta_{i 1} \mid a_{i 1}, y_{i}\right)$ (which is identified from the previous paragraph), ${ }^{21}$ the densities $f\left(c_{1} \mid a_{1}, \eta_{1}, y_{1}\right)$ and $f\left(c_{1}, \eta_{1} \mid a_{1}, y\right)$ are thus identified. Identification of the consumption function (10) for $t=1$ follows since $g_{1}$ is the conditional quantile function of $c_{1}$ given $a_{1}, \eta_{1}$, and $\varepsilon_{1}=y_{1}-\eta_{1}$.

Second Period's Assets. Turning to period 2, we have, using Assumption 1(i) and (iii),

$$
f\left(a_{2} \mid c_{1}, a_{1}, y\right)=\int f\left(a_{2} \mid c_{1}, a_{1}, \eta_{1}, y_{1}\right) f\left(\eta_{1} \mid c_{1}, a_{1}, y\right) d \eta_{1},
$$

from which it follows that the density $f\left(a_{2} \mid c_{1}, a_{1}, \eta_{1}, y_{1}\right)$ is identified, provided the distribution of $\left(\eta_{i 1} \mid c_{i 1}, a_{i 1}, y_{i}\right)$ (which is identified from above) is complete in $\left(y_{i 2}, \ldots, y_{i T}\right)$.

In addition, using Bayes's rule and Assumption 1(i) and (iii),

$$
f\left(\eta_{2} \mid a_{2}, c_{1}, a_{1}, y\right)=\int \frac{f\left(y \mid \eta_{1}, \eta_{2}, y_{1}\right) f\left(\eta_{1}, \eta_{2} \mid a_{2}, c_{1}, a_{1}, y_{1}\right)}{f\left(y \mid a_{2}, c_{1}, a_{1}, y_{1}\right)} d \eta_{1} .
$$

So, as the density $f\left(\eta_{1} \mid a_{2}, c_{1}, a_{1}, y_{1}\right)$ is identified from above, and by Assumption 1

$$
f\left(\eta_{1}, \eta_{2} \mid a_{2}, c_{1}, a_{1}, y_{1}\right)=f\left(\eta_{1} \mid a_{2}, c_{1}, a_{1}, y_{1}\right) f\left(\eta_{2} \mid \eta_{1}\right),
$$

it follows that $f\left(\eta_{2} \mid a_{2}, c_{1}, a_{1}, y\right)$ is identified.

Subsequent Periods. To see how the argument extends to subsequent periods, consider second period's consumption. We have, using Assumption 1(iii),

$$
f\left(c_{2} \mid a_{2}, c_{1}, a_{1}, y\right)=\int f\left(c_{2} \mid a_{2}, \eta_{2}, y_{2}\right) f\left(\eta_{2} \mid a_{2}, c_{1}, a_{1}, y\right) d \eta_{2} .
$$

Provided the distribution of $\left(\eta_{i 2} \mid a_{i 2}, c_{i 1}, a_{i 1}, y_{i}\right)$ (which is identified from the previous paragraph) is complete in $\left(c_{i 1}, a_{i 1}, y_{i 1}, y_{i 3}, \ldots, y_{i T}\right)$, the density $f\left(c_{2} \mid a_{2}, \eta_{2}, y_{2}\right)$ is identified.

By induction, using in addition Assumption 1(ii) from the third period onward, the joint density of $\eta$ 's, consumption, assets, and earnings is identified provided, for all $t \geq 1$, the distributions of $\left(\eta_{i t} \mid c_{i}^{t}, a_{i}^{t}, y_{i}\right)$ and $\left(\eta_{i t} \mid c_{i}^{t-1}, a_{i}^{t}, y_{i}\right)$ are complete in $\left(c_{i}^{t-1}, a_{i}^{t-1}, y_{i}^{t-1}, y_{i, t+1}, \ldots, y_{i T}\right)$.

Discussion. The identification arguments depend on completeness conditions, which relate to the relevance, in a nonparametric sense, of the instruments. To illustrate this, consider the completeness of the distribution of $\left(\eta_{i 1} \mid y_{i}\right)$ in $\left(y_{i 2}, \ldots, y_{i T}\right)$, which we use to show the identification of the consumption rule in the first period; see (16). Here we abstract from assets for simplicity. The completeness condition then depends on the properties of the earnings process. As an example, consider the case where $T=2$, and $\left(\eta_{i 1}, y_{i 1}, y_{i 2}\right)$ follows a multivariate normal distribution with zero mean. Then $\eta_{i 1}=\alpha y_{i 1}+\beta y_{i 2}+\zeta_{i}$, where $\zeta_{i}$ is normal $\left(0, \sigma^{2}\right)$, independent of $\left(y_{i 1}, y_{i 2}\right)$. It can be easily shown that $\beta \neq 0$ if $\operatorname{Cov}\left(\eta_{i 1}, \eta_{i 2}\right) \neq 0$, in which case the distribution of $\left(\eta_{i 1} \mid y_{i 1}, y_{i 2}\right)$ is complete in $y_{i 2}$. As in the identification of the earnings process, identification of the consumption rule thus relies on $\eta$ 's being dependent over time. Beyond the settings studied so far in the literature

\footnotetext{
${ }^{21}$ Here, by completeness in $y_{i 2}$ of the distribution of $\left(y_{i 1} \mid y_{i 2}, x_{i}\right)$ we mean that the only solution to $\mathbb{E}\left[h\left(y_{i 1}, x_{i}\right) \mid y_{i 2}, x_{i}\right]=0$ is $h=0$. This is the same as $\left(y_{i 1}, x_{i}\right) \mid\left(y_{i 2}, x_{i}\right)$ being complete. Note that, similarly as before, the weaker condition of bounded completeness suffices.
} 
(such as in D'Haultfoeuille (2011), Andrews (2011), or Hu and Shiu (2012)), it would be of great interest to provide primitive conditions for completeness in the nonlinear models we focus on here.

An intuitive explanation for the identification argument comes from the link to nonparametric instrumental variables (NPIV). In period 1 , for a fixed $a_{1},(14)$ is analogous to an NPIV problem where $\eta_{i 1}$ is the endogenous regressor and $y_{i}=\left(y_{i 1}, \ldots, y_{i T}\right)$ is the vector of instruments. Likewise, conditional on $\left(a_{i 1}, y_{i 1}\right),\left(y_{i 2}, \ldots, y_{i T}\right)$ are the "excluded instruments" for $\eta_{i 1}$ in (16). In subsequent periods, lagged consumption and assets are used as instruments, together with lags and leads of earnings. Using leads of log-earnings for identifying consumption responses is a common strategy in linear models; see, for example, Hall and Mishkin (1982) and Blundell, Pistaferri, and Preston (2008).

\subsection{Household Unobserved Heterogeneity}

Accounting for unobserved heterogeneity in preferences or discounting, for example, may be empirically important. Heterogeneity in discount factors is also a popular mechanism in quantitative macroeconomic models to generate realistic wealth inequality; see, for example, Krusell and Smith (1998) and Krueger, Mitman, and Perri (2015). With unobserved heterogeneity, the consumption rule takes the form

$$
c_{i t}=g_{t}\left(a_{i t}, \eta_{i t}, \varepsilon_{i t}, \xi_{i}, \widetilde{\nu}_{i t}\right), \quad t=1, \ldots, T,
$$

where $\xi_{i}$ is a household-specific effect and $\widetilde{\nu}_{i t}$ are i.i.d. standard uniform. The distribution of $\left(\xi_{i}, \eta_{i 1}, a_{i 1}\right)$ is left unrestricted. Therefore, $\xi_{i}$ is treated as a "fixed effect." Even if a fully unstructured distinction between unobserved heterogeneity and individual dynamics in a finite horizon panel is not possible, finite-dimensional fixed effects can be included nonparametrically in the consumption (and earnings) equations as long as $T$ is sufficiently large. In Appendix A, we provide conditions for identification of this model, by relying on results from $\mathrm{Hu}$ and Schennach (2008). For simplicity, we consider scalar heterogeneity $\xi_{i}$. Depending on the number of available time periods, a vector of unobserved heterogeneity could be allowed for.

Last, in Section S3 of the Supplemental Material, we consider several extensions of the model. We show how to allow for unobserved heterogeneity in earnings, as in the following specification that we will take to the data:

$$
y_{i t}=\eta_{i t}+\zeta_{i}+\varepsilon_{i t},
$$

where $\eta_{i t}=Q_{t}\left(\eta_{i, t-1}, u_{i t}\right)$ is first-order Markov. We also describe how to allow for dependence in transitory shocks, advance earnings information, and consumption habits.

\section{ESTIMATION STRATEGY}

\subsection{Empirical Specification}

Earnings Components. The earnings model depends on the Markovian transitions of the persistent component $Q_{t}(\cdot, \cdot)$, the marginal distributions of $\varepsilon_{i t}$, and the marginal distribution of the initial persistent component $\eta_{i 1}$. We now explain how we empirically specify these three components. 
Let $\varphi_{k}$, for $k=0,1, \ldots$, denote a dictionary of bivariate functions, with $\varphi_{0}=1$. Letting $a g e_{i t}$ denote the age of the head of household $i$ in period $t$, we specify

$$
\begin{aligned}
Q_{t}\left(\eta_{i, t-1}, \tau\right) & =Q\left(\eta_{i, t-1}, \text { age }_{i t}, \tau\right) \\
& =\sum_{k=0}^{K} a_{k}^{Q}(\tau) \varphi_{k}\left(\eta_{i, t-1}, \text { age }_{i t}\right) .
\end{aligned}
$$

In practice, we use low-order products of Hermite polynomials for $\varphi_{k}$. We specify the quantile function of $\varepsilon_{i t}$ (for $t=1, \ldots, T$ ) given age $e_{i t}$, and that of $\eta_{i 1}$ given age at the start of the period $a g e_{i 1}$, in a similar way. Specifically, we set

$$
\begin{gathered}
Q_{\varepsilon}\left(\text { age }_{i t}, \tau\right)=\sum_{k=0}^{K} a_{k}^{\varepsilon}(\tau) \varphi_{k}\left(\text { age }_{i t}\right), \\
Q_{\eta_{1}}\left(\text { age }_{i 1}, \tau\right)=\sum_{k=0}^{K} a_{k}^{\eta_{1}}(\tau) \varphi_{k}\left(\text { age }_{i 1}\right),
\end{gathered}
$$

with outcome-specific choices for $K$ and $\varphi_{k}$.

The quantile model (21) provides a flexible specification of the conditional distribution of $\eta_{i t}$ given $\eta_{i, t-1}$ and age. Similarly, our quantile specifications flexibly model how $\varepsilon_{i t}$ and $\eta_{i 1}$ depend on age, at every quantile. We include the age of the household head as a control, while ruling out dependence on calendar time. This choice is motivated by our desire to model life-cycle evolution, as well as by the relative stationarity of the earnings distributions (conditional on age) during the 1999-2009 period and the relatively small sample size. On larger samples, an interesting avenue will be to allow for variation in both age and calendar time within our framework. ${ }^{22}$

Consumption Rule. Following from Section 3.2, we specify the conditional distribution of consumption given current assets and earnings components as follows:

$$
\begin{aligned}
g_{t}\left(a_{i t}, \eta_{i t}, \varepsilon_{i t}, \tau\right) & =g\left(a_{i t}, \eta_{i t}, \varepsilon_{i t}, a g e_{i t}, \tau\right) \\
& =\sum_{k=1}^{K} b_{k}^{g} \widetilde{\varphi}_{k}\left(a_{i t}, \eta_{i t}, \varepsilon_{i t}, a g e_{i t}\right)+b_{0}^{g}(\tau),
\end{aligned}
$$

where $\widetilde{\varphi}_{k}$ is a dictionary of functions (in practice, another product of Hermite polynomials).

Equation (22) is a nonlinear regression model. In contrast with (21), the consumption model is additive in $\tau$. It would be conceptually straightforward to let all coefficients $b_{k}^{g}$ depend on $\tau$, although this would lead to a less parsimonious specification. Below we augment the consumption function to also depend nonlinearly on a household-specific effect.

\footnotetext{
${ }^{22}$ The functional form in (21) does not enforce monotonicity in $\tau$, but our estimation method will produce an automatic rearrangement of quantiles if needed.
} 
Assets Evolution. We specify the distribution of initial assets $a_{i 1}$ conditional on the initial persistent component $\eta_{i 1}$ and the age at the start of the period age ${ }_{i 1}$ as

$$
Q_{a}\left(\eta_{i 1}, \text { age }_{i 1}, \tau\right)=\sum_{k=0}^{K} b_{k}^{a}(\tau) \widetilde{\varphi}_{k}\left(\eta_{i 1}, \text { age }_{i 1}\right),
$$

for different choices for $K$ and $\widetilde{\varphi}_{k}$. We then specify how assets evolve as a function of lagged assets, consumption, earnings, the persistent earnings component $\eta$, and age, using (11), where

$$
\begin{aligned}
h_{t}\left(a_{i, t-1}, c_{i, t-1}, y_{i, t-1}, \eta_{i, t-1}, \tau\right) & =h\left(a_{i, t-1}, c_{i, t-1}, y_{i, t-1}, \eta_{i, t-1}, \operatorname{age}_{i t}, \tau\right) \\
& =\sum_{k=1}^{K} b_{k}^{h} \widetilde{\varphi}_{k}\left(a_{i, t-1}, c_{i, t-1}, y_{i, t-1}, \eta_{i, t-1}, \text { age }_{i t}\right)+b_{0}^{h}(\tau),
\end{aligned}
$$

for some $K$ and $\widetilde{\varphi}_{k} \cdot 23$

Implementation. The functions $a_{k}^{Q}, a_{k}^{\varepsilon}$, and $a_{k}^{\eta_{1}}$ are indexed by a finite-dimensional parameter vector $\theta$. Likewise, the functions $b_{0}^{g}, b_{0}^{h}$, and $b_{k}^{a}$ are indexed by a parameter vector $\mu$ that also contains $b_{1}^{g}, \ldots, b_{K}^{g}, b_{1}^{h}, \ldots, b_{K}^{h}$. We base our implementation on Wei and Carroll (2009) and Arellano and Bonhomme (2016). As in these papers, we model the functions $a_{k}^{Q}$ as piecewise-polynomial interpolating splines on a grid $\left[\tau_{1}, \tau_{2}\right],\left[\tau_{2}, \tau_{3}\right], \ldots$, $\left[\tau_{L-1}, \tau_{L}\right]$, contained in the unit interval. We extend the specification of the intercept coefficient $a_{0}^{Q}$ on $\left(0, \tau_{1}\right]$ and $\left[\tau_{L}, 1\right)$ using a parametric model indexed by $\lambda^{Q}$. All $a_{k}^{Q}$ for $k \geq 1$ are constant on $\left[0, \tau_{1}\right]$ and $\left[\tau_{L}, 1\right]$, respectively. Hence, denoting $a_{k \ell}^{Q}=a_{k}^{Q}\left(\tau_{\ell}\right)$, the functions $a_{k}^{Q}$ depend on $\left\{a_{11}^{Q}, \ldots, a_{K L}^{Q}, \lambda^{Q}\right\}$.

Unlike in an ordinary quantile regression, the dependence of the parameters on the percentiles $\tau$ needs to be specified because some of our regressors are latent variables. In practice, we take $L=11$ and $\tau_{\ell}=\ell /(L+1)$. The functions $a_{k}^{Q}$ are taken as piecewiselinear on $\left[\tau_{1}, \tau_{L}\right]$. An advantage of this specification is that the likelihood function is available in closed form. In addition, we specify $a_{0}^{Q}$ as the quantile of an exponential distribution on $\left(0, \tau_{1}\right]$ (with parameter $\lambda_{-}^{Q}$ ) and $\left[\tau_{L}, 1\right)$ (with parameter $\left.\lambda_{+}^{Q}\right){ }^{24}$

We proceed similarly to model $a_{k}^{\varepsilon}, a_{k}^{\eta_{1}}$, and $b_{k}^{a}$. Moreover, as our data show little evidence against consumption being log-normal, we set $b_{0}^{g}(\tau)$ to $\alpha+\sigma \Phi^{-1}(\tau)$, where $(\alpha, \sigma)$ are parameters to be estimated. We proceed similarly for $b_{0}^{h}(\tau)$. We also estimated two different versions of the model with more flexible specifications for $b_{0}^{g}(\tau)$ and $b_{0}^{h}(\tau)$ : based on quantiles on a grid with $L=11$ knots, and allowing for an age effect in the variance of the consumption innovation. In both cases, we found very similar results to the ones

\footnotetext{
${ }^{23}$ In a previous version of the paper, we estimated the model imposing that $\eta_{i, t-1}$ does not enter (24), which is still consistent with the budget constraint (7) and avoids the need to model predetermined assets. We obtained qualitatively similar empirical results.

${ }^{24}$ As a result, we have
}

$$
\begin{aligned}
a_{0}^{Q}(\tau)= & \frac{1}{\lambda_{-}^{Q}} \log \left(\frac{\tau}{\tau_{1}}\right) \mathbf{1}\left\{0<\tau<\tau_{1}\right\}+\sum_{\ell=1}^{L-1}\left(a_{k \ell}^{Q}+\frac{a_{k, \ell+1}^{Q}-a_{k \ell}^{Q}}{\tau_{\ell+1}-\tau_{\ell}}\left(\tau-\tau_{\ell}\right)\right) \mathbf{1}\left\{\tau_{\ell} \leq \tau<\tau_{\ell+1}\right\} \\
& -\frac{1}{\lambda_{+}^{Q}} \log \left(\frac{1-\tau}{1-\tau_{L}}\right) \mathbf{1}\left\{\tau_{L} \leq \tau<1\right\} .
\end{aligned}
$$


we report below. We use tensor products of Hermite polynomials for $\varphi_{k}$ and $\widetilde{\varphi}_{k}$, each component of the product taking as argument a standardized variable. ${ }^{25}$

Household Unobserved Heterogeneity. When allowing for household unobserved heterogeneity in consumption/assets, we model log-consumption as

$$
c_{i t}=g\left(a_{i t}, \eta_{i t}, \varepsilon_{i t}, \text { age }_{i t}, \xi_{i}, \widetilde{\nu}_{i t}\right),
$$

which we specify similarly as in (22), with parameters $\widetilde{b}_{k}^{g}$. To fix the scale of the function, we impose that

$$
\sum_{k=1}^{K} \widetilde{b}_{k}^{g} \widetilde{\varphi}_{k}(0,0,0, \overline{a g e}, \xi)=\xi \text { for all } \xi,
$$

where $\overline{a g e}$ denotes the mean value of age in the sample. Likewise, we model assets as

$$
a_{i t}=h\left(a_{i, t-1}, c_{i, t-1}, y_{i, t-1}, \eta_{i, t-1}, \operatorname{age}_{i t}, \xi_{i}, \widetilde{v}_{i t}\right),
$$

with a similar specification as in (24). Last, we specify $\xi_{i}=q\left(a_{i 1}, \eta_{i 1}, a g e_{i 1}, \omega_{i}\right)$, with $\omega_{i}$ uniform on $(0,1)$, using a quantile modeling as in $(23) .{ }^{26}$

\subsection{Overview of the Estimation Algorithm}

The algorithm is an adaptation of techniques developed in Arellano and Bonhomme (2016) to a setting with time-varying latent variables. The first estimation step recovers estimates of the earnings parameters $\theta$. The second step recovers estimates of the consumption and assets parameters $\mu$, given a previous estimate of $\theta$. Our choice of a sequential estimation strategy, rather than joint estimation of $(\theta, \mu)$, is motivated by the fact that $\theta$ is identified from the earnings process alone. In contrast, in a joint estimation approach, estimates of the earnings process would be partly driven by the consumption model. Here we describe the estimation of the earnings parameters $\theta$. Estimation of the consumption parameters $\mu$ is similar. The model's restrictions are described in detail in Appendix B.

A compact notation for the restrictions implied by the earnings model is

$$
\bar{\theta}=\underset{\theta}{\operatorname{argmin}} \mathbb{E}\left[\int R\left(y_{i}, \eta ; \theta\right) f_{i}(\eta ; \bar{\theta}) d \eta\right],
$$

where $R$ is a known function, $\bar{\theta}$ denotes the true value of $\theta$, and $f_{i}(\cdot ; \bar{\theta})=f\left(\cdot \mid y_{i}^{T}\right.$, age $\left.e_{i}^{T} ; \bar{\theta}\right)$ denotes the posterior density of $\left(\eta_{i 1}, \ldots, \eta_{i T}\right)$ given the earnings data. The estimation algorithm is closely related to the "stochastic EM" algorithm (Celeux and Diebolt (1993)). Stochastic EM is a simulated version of the classical EM algorithm of Dempster, Laird, and Rubin (1977), where new draws from $\eta$ are computed in every iteration of the algorithm. One difference is that, unlike in EM, our problem is not likelihood-based. Instead, we exploit the computational convenience of quantile regression and replace likelihood maximization by a sequence of quantile regressions in each $M$-step of the algorithm.

Starting with a parameter vector $\widehat{\theta}^{(0)}$, we iterate the following two steps on $s=0,1,2, \ldots$ until convergence of the $\widehat{\theta}^{(s)}$ process:

\footnotetext{
${ }^{25}$ For example, $a_{t} / \operatorname{std}(a), \eta_{t} / \operatorname{std}(y), \varepsilon_{t} / \operatorname{std}(y)$, and (age $e_{t}-$ mean (age)) $/ \operatorname{std}($ age $)$ are used as arguments of the consumption rule.

${ }^{26} \mathrm{We}$ proceed analogously when allowing for an additive household-specific effect $\zeta_{i}$ in log-earnings $y_{i t}=$ $\eta_{i t}+\zeta_{i}+\varepsilon_{i t}$, where $\eta_{i t}$ is given by (2). There we allow for flexible dependence between $\eta_{i 1}$, $\zeta_{i}$, and $a g e_{i 1}$ through another series quantile model.
} 
1. Stochastic E-step: Draw $\eta_{i}^{(m)}=\left(\eta_{i 1}^{(m)}, \ldots, \eta_{i T}^{(m)}\right)$ for $m=1, \ldots, M$ from $f_{i}\left(\cdot ; \widehat{\theta}^{(s)}\right)$.

2. M-step: Compute

$$
\widehat{\theta}^{(s+1)}=\underset{\theta}{\operatorname{argmin}} \sum_{i=1}^{N} \sum_{m=1}^{M} R\left(y_{i}, \eta_{i}^{(m)} ; \theta\right) .
$$

Note that, as the likelihood function is available in closed form, the E-step is straightforward. In practice, we use a random-walk Metropolis-Hastings sampler for this purpose, targeting an acceptance rate of approximately $30 \%$. The M-step consists of a number of quantile regressions. For example, the parameters $a_{k \ell}^{Q}$ are updated as

$$
\min _{\left(a_{0 \ell}^{Q}, \ldots, a_{K \ell}^{Q}\right.} \sum_{i=1}^{N} \sum_{t=2}^{T} \sum_{m=1}^{M} \rho_{\tau \ell}\left(\eta_{i t}^{(m)}-\sum_{k=0}^{K} a_{k \ell}^{Q} \varphi_{k}\left(\eta_{i, t-1}^{(m)}, a g e_{i t}\right)\right), \quad \ell=1, \ldots, L,
$$

where $\rho_{\tau}(u)=u(\tau-\mathbf{1}\{u \leq 0\})$ is the "check" function. This is a set of standard quantile regressions, associated with convex objective functions. We proceed in a similar way to update all other parameters; see Appendix B for details.

In practice, we first estimate the effect of age on mean log-earnings by regressing them on a quartic in age. We then impose in each iteration of the algorithm that $\varepsilon_{i t}$ and age are uncorrelated (although we allow for age effects on the variance and quantiles of $\varepsilon_{i t}$ ). We take $M=1$, stop the chain after a large number of iterations, and report an average across the last $\widetilde{S}$ values $\widehat{\theta}=\frac{1}{S} \sum_{s=S-S}^{S} \widetilde{S}_{+1} \widehat{\theta}^{(s)}$, and similarly for consumption-related parameters $\widehat{\mu} .{ }^{27}$ The results for the earnings parameters are based on $S=500$ iterations, with 200 Metropolis-Hastings draws in each iteration. Consumption-related parameters are estimated using 200 iterations with 200 draws per iteration. In both cases, we take $\widetilde{S}=S / 2$. In our experiments, we observed that the algorithm may get "stuck" on what appears to be a local regime of the Markov chain. We started the algorithm from a large number of initial parameter values, and selected the estimates yielding the highest average log-likelihood over iterations. The non-selected values tended to give very similar pictures to the ones we report below.

Properties. Nielsen (2000) studied the statistical properties of the stochastic EM algorithm in a likelihood case. He provided conditions under which the Markov chain $\widehat{\theta}^{(s)}$ is ergodic, for a fixed sample size. He also characterized the asymptotic distribution of $\widehat{\theta}$ as the sample size $N$ tends to infinity. Arellano and Bonhomme (2016) characterized the asymptotic distribution of $\widehat{\theta}$ in a case where the optimization step is not likelihood-based but relies on quantile-based estimating equations. The estimator $\widehat{\theta}$ is root- $N$ consistent and asymptotically normal under correct specification of the parametric model, for $K$ and $L$ fixed. Note that an alternative, nonparametric approach would be to let $K$ and $L$ increase with $N$ at an appropriate rate so as to let the approximation bias tend to zero. ${ }^{28}$ Studying inference in our problem as $(N, K, L)$ jointly tend to infinity is an interesting avenue for future work.

\footnotetext{
${ }^{27}$ As an alternative to taking a large $M$, taking $M=1$ and averaging the parameter draws is computationally convenient in our setting.

${ }^{28}$ See Belloni, Chernozhukov, Chetverikov, and Fernández-Val (2016) for an analysis of inference for series quantile regression, and Arellano and Bonhomme (2016) for a consistency analysis in a panel data model closely related to the one we consider here.
} 


\section{EMPIRICAL RESULTS ON EARNINGS AND CONSUMPTION}

In this section, we present our empirical results. We start by describing our main data source, the Panel Study of Income Dynamics (PSID). We then show how earnings and consumption respond to income shocks. We also corroborate our findings for the nonlinear earnings process using administrative data on household earnings from the Norwegian population register. Finally, we report simulation exercises based on the estimated model.

\subsection{Panel Data}

Panel data on household consumption, income, and assets are rare. The PSID began the collection of detailed data on consumption expenditures and asset holdings in 1999, in addition to household earnings and demographics. An annual wave is available every other year. We use data for the 1999-2009 period (six waves).

Earnings $Y_{i t}$ are total pre-tax household labor earnings. We construct $y_{i t}$ as residuals from regressing log household earnings on a set of demographics, which include cohort interacted with education categories for both household members, race, state, and largecity dummies, a family size indicator, number of kids, a dummy for income recipient other than husband and wife, and a dummy for kids out of the household. Controls for family size and composition are included so as to equivalize household earnings (likewise for consumption and assets below). Education, race, and geographic dummies are included in an attempt to capture individual heterogeneity beyond cohort effects and the initial persistent component of earnings $\eta_{i 1}$. Removing demographic-specific means in a preliminary step has been the standard practice in the empirical analysis of earnings dynamics. A more satisfactory approach would integrate both steps, especially given our emphasis on nonlinearities. However, except for age, we did not attempt a richer conditioning in light of sample size.

We use data on consumption $C_{i t}$ of nondurables and services. The panel data contain information on health expenditures, utilities, car-related expenditures and transportation, education, child care, and food expenditures. Recreation, alcohol, tobacco, and clothing (the latter available from 2005) are the main missing items. Rent information is available for renters, but not for home owners. We follow Blundell, Pistaferri, and Saporta-Eksten (2016) and impute rent expenditures for home owners. ${ }^{29}$ In total, approximately $67 \%$ of consumption expenditures on nondurables and services are covered. We construct $c_{i t}$ as residuals of log total consumption on the same set of demographics as for earnings.

Asset holdings $A_{i t}$ are constructed as the sum of financial assets (including cash, stocks, and bonds), real estate value, pension funds, and car value, net of mortgages and other debt. We construct residuals $a_{i t}$ by regressing log-assets on the same set of demographics as for earnings and consumption. These log-assets residuals will enter as arguments of the nonlinear consumption rule (10).

To select the sample, we follow Blundell, Pistaferri, and Saporta-Eksten (2016) and focus on a sample of participating and married male heads aged between 25 and 60 . We drop all observations for which data on earnings, consumption, or assets, either in levels or log-residuals, are missing. See Appendix C for further details. In the analysis, we focus on a balanced subsample of $N=792$ households. Table C.I in Appendix C shows mean total earnings, consumption, and asset holdings, by year. Compared to Blundell, Pistaferri, and Saporta-Eksten (2016), households in our balanced sample have higher assets,

\footnotetext{
${ }^{29}$ Note that, as a result, consumption responds automatically to variations in house prices. An alternative would be to exclude rents and imputed rents from consumption expenditures.
} 
and to a lesser extent higher earnings and consumption. The table also shows a large and increasing dispersion of assets across households. The evolution of assets may partly reflect the housing boom and bust, including the effect of the Great Recession at the end of the sample. Although our framework could be used to document distributional dynamics along the business-cycle, we abstract from business-cycle effects in this paper.

Last, the sample that we use is relatively homogeneous. Including households with less stable employment histories would be interesting, but it would require extending our framework. We return to this point in the conclusion.

\subsection{Earnings}

We next comment on the empirical estimates of the earnings process. Figure 2(a) reproduces Figure 1(a). It shows estimates of the average derivative of the conditional quantile function of log-earnings residuals $y_{i t}$ given $y_{i, t-1}$ with respect to $y_{i, t-1}$ in the PSID sample. The figure suggests the presence of nonlinear persistence, which depends on both the percentile of past income $\left(\tau_{\text {init }}\right)$ and the percentile of the quantile innovation $\left(\tau_{\text {shock }}\right)$. This empirical pattern is also present for male wages; see Figure S1 of the Supplemental Material. We then estimate the earnings model, ${ }^{30}$ and, given the estimated parameters, we simulate the model..$^{31}$ Figure 2(b), which is based on simulated data, shows that our nonlinear model reproduces the patterns of nonlinear persistence well. In contrast, standard models have difficulty fitting this empirical pattern. For example, we estimated a simple version of the canonical earnings dynamics model (3) with a random-walk component and independent transitory shocks. ${ }^{32}$ Figure 2(c) shows that the average derivative of the quantile function is nearly constant (up to simulation error) with respect to $\tau_{\text {shock }}$ and $\tau_{\text {init }}$. This linear specification without interaction effects between earnings shocks and past earnings components stands in contrast with the data. ${ }^{33}$

Figure 2(d) then shows the estimated persistence of the earnings component $\eta_{i t}$. Specifically, the graph shows $\rho_{t}\left(\eta_{i, t-1}, \tau\right)$ from equation (4), evaluated at percentiles $\tau_{\text {init }}$ and $\tau_{\text {shock }}$ and at the mean age in the sample (47.5 years).$^{34}$ Persistence in $\eta$ 's is higher than persistence in log-earnings residuals, consistently with the fact that Figure 2(d) is net of transitory shocks. Persistence is close to 1 for high-earnings households hit by good shocks, and for low-earnings households hit by bad shocks. At the same time, persistence is lower, down to $0.6-0.8$, when bad shocks hit high-earnings households or good shocks hit low-earnings ones.

Densities and Moments. Figure 3 shows estimates of the marginal distributions of the persistent and transitory earnings components at mean age. While the persistent component $\eta_{i t}$ shows relatively small departures from Gaussianity, the density of $\varepsilon_{i t}$ is clearly

\footnotetext{
${ }^{30}$ We use tensor products of Hermite polynomials of degrees $(3,2)$ for the conditional quantile function of $\eta_{i t}$ given $\eta_{i, t-1}$ and age, and second-order polynomials for $\varepsilon_{i t}$ and $\eta_{i 1}$ as a function of age.

${ }^{31}$ We draw 20 earnings values per household. In the simulation, we impose that the support of simulated $\eta$ draws be less than three times the empirical support of log-earnings residuals. This affects very few observations.

${ }^{32}$ Estimation is based on equally-weighted minimum distance using the covariance structure predicted by the canonical model.

${ }^{33}$ In order to assess the sensitivity to the polynomial functional form that we use, in Figure S2 of the Supplemental Material, we report persistence estimates based on piecewise-linear specifications based on 9 or 25 pieces. The characteristic shape of Figure 2(a) remains present.

${ }^{34}$ The estimated persistence is similar when averaging over age; see Figure S3 of the Supplemental Material.
} 
(a) Earnings, PSID data

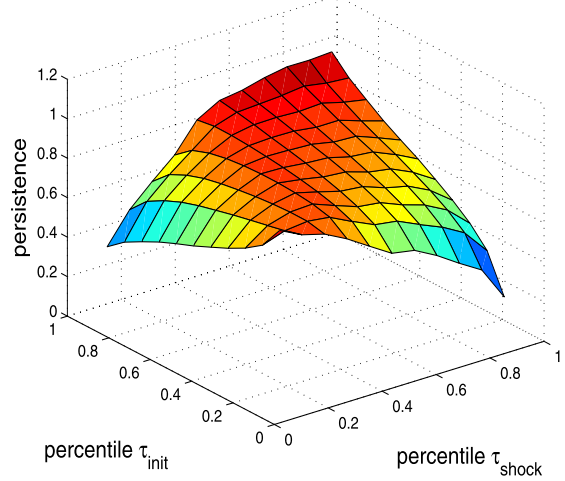

(c) Earnings, canonical model

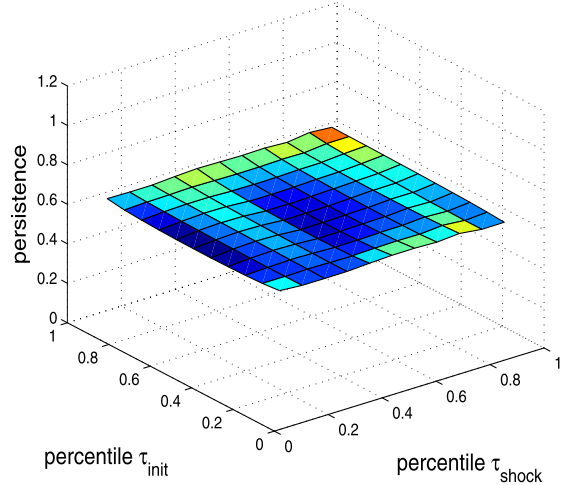

(b) Earnings, nonlinear model

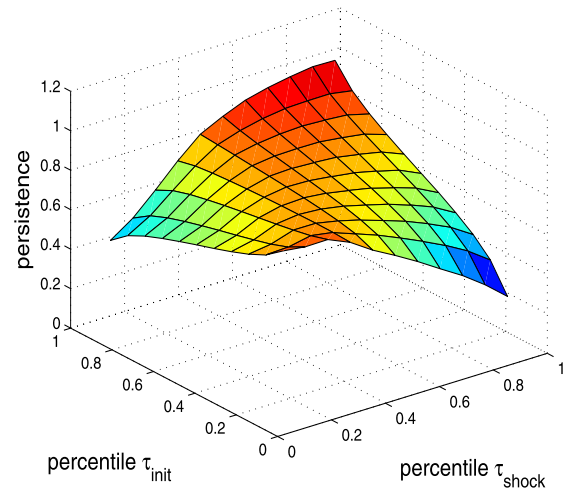

(d) Persistent component $\eta_{i t}$, nonlinear model

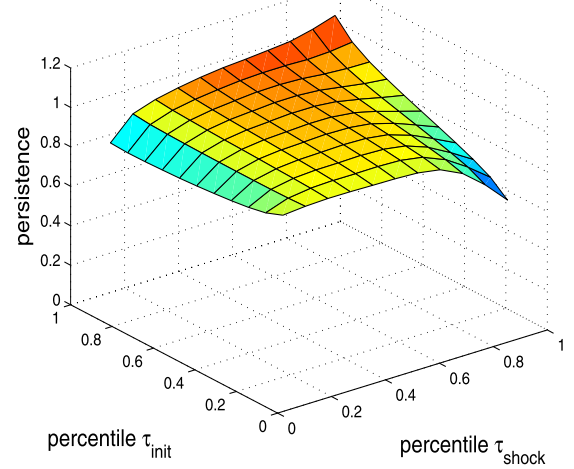

FIGURE 2.-Nonlinear persistence. Note: Graphs (a), (b), and (c) show estimates of the average derivative of the conditional quantile function of $y_{i t}$ given $y_{i, t-1}$ with respect to $y_{i, t-1}$, evaluated at percentile $\tau_{\text {shock }}$ and at a value of $y_{i, t-1}$ that corresponds to the $\tau_{\text {init }}$ percentile of the distribution of $y_{i, t-1}$. Graph (a) is based on the PSID data, graph (b) is based on data simulated according to our nonlinear earnings model with parameters set to their estimated values, and graph (c) is based on data simulated according to the canonical random-walk earnings model (3). Graph (d) shows estimates of the average derivative of the conditional quantile function of $\eta_{i t}$ on $\eta_{i, t-1}$ with respect to $\eta_{i, t-1}$, based on estimates from the nonlinear earnings model.

non-normal and presents high kurtosis and fat tails. These results are qualitatively consistent with empirical estimates of non-Gaussian linear models in Horowitz and Markatou (1996) and Bonhomme and Robin (2010).

In Figure 4, we report the measure of conditional skewness in (6), for $\tau=11 / 12$, for both log-earnings residuals (left graph) and the $\eta$ component (right). Panel (b) shows that $\eta_{i t}$ is positively skewed for low values of $\eta_{i, t-1}$, and negatively skewed for high values of $\eta_{i, t-1}$. This is in line with the nonlinear persistence reported in Figure 2(d): when low- $\eta$ households are hit by an unusually positive shock, dependence of $\eta_{i t}$ on $\eta_{i, t-1}$ is low with the result that they have a relatively large probability of outcomes far to the right from the central part of the distribution. Likewise, high- $\eta$ households have a relatively large probability of getting outcomes far to the left of their distribution associated with low 
(a) Persistent component $\eta_{i t}$

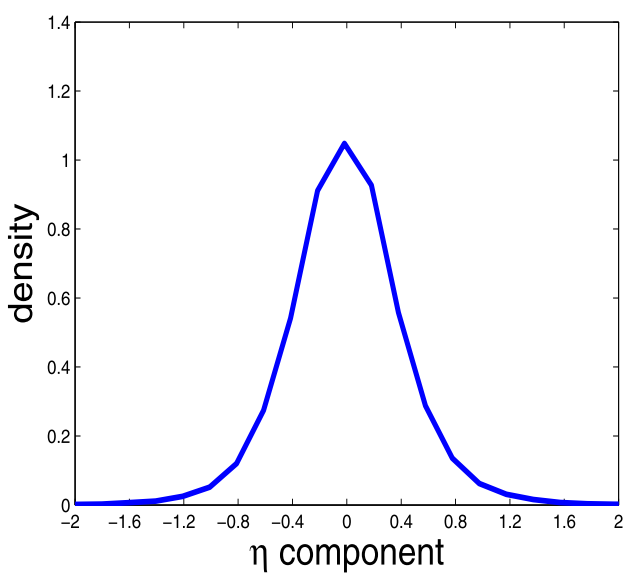

(b) Transitory component $\varepsilon_{i t}$

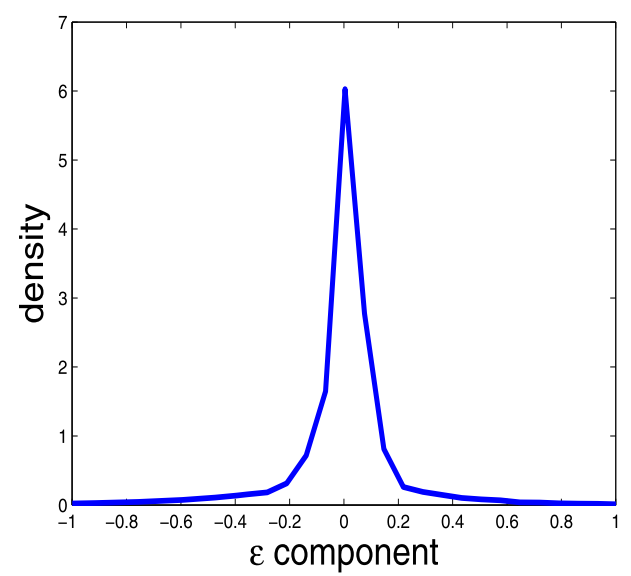

FIGURE 3.-Densities of persistent and transitory earnings components. Note: Nonparametric estimates of densities based on simulated data according to the nonlinear model, using a Gaussian kernel.

persistence episodes. Panel (a) similarly suggests the presence of conditional asymmetry in log-earnings residuals, although the evidence seems less strong than for $\eta$.

In addition, in Figures S4 to S8 of the Supplemental Material, we report several measures of fit of the model. We show quantile-based estimates of conditional dispersion and conditional skewness. We also report estimates of the skewness, kurtosis, and densities of log-earnings residuals growth at various horizons, from 2 to 10 years. The data suggest the presence of ARCH effects (as in Meghir and Pistaferri (2004)). It also shows that log-earnings growth is non-Gaussian, displaying negative skewness and high kurtosis. Guvenen et al. (2015) documented similar features on U.S. administrative data. This shows both the qualitative similarity between the PSID and the administrative U.S. data

(a) Log-earnings residuals $y_{i t}$

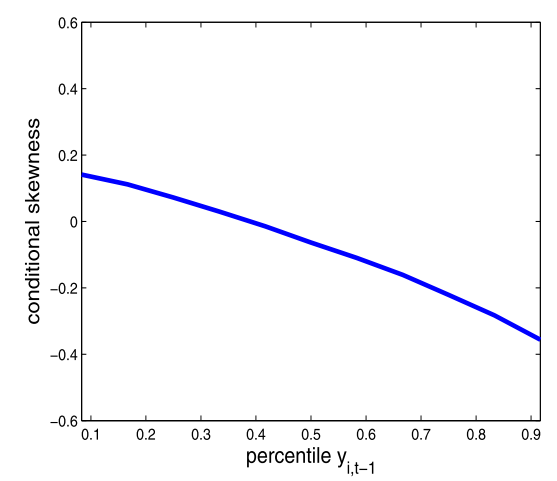

(b) Persistent component $\eta_{i t}$

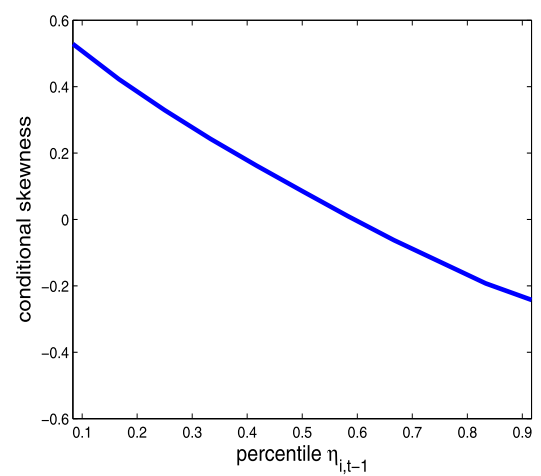

FIGURE 4.-Conditional skewness of log-earnings residuals and $\eta$ component. Note: Conditional skewness $s k(y, \tau)$ and $s k(\eta, \tau)$, see equation (6), for $\tau=11 / 12$. Log-earnings residuals (data, left) and $\eta$ component (right). The $x$-axis shows the conditioning variable; the $y$-axis shows the corresponding value of the conditional skewness measure. 
in terms of higher moments of log-earnings growth, and the ability of our nonlinear model to fit these features. Note that, in our model, skewness and excess kurtosis of log-earnings growth at long horizons are mostly due to the non-Gaussianity of the transitory component $\varepsilon$.

Confidence Bands. We compare two different methods to compute confidence intervals for these estimates. The first method is the nonparametric bootstrap, clustered at the household level. The second method is the parametric bootstrap. While the latter requires correct specification of the parametric model, the former may still be consistent under misspecification and allows for unrestricted serial correlation (however, we are not aware of a formal justification for it in this setting). In Figures S9 to S14 of the Supplemental Material, we report pointwise $95 \%$ confidence bands based on both methods, and we also show uniform confidence bands based on the nonparametric bootstrap. In all cases, the main findings on nonlinear persistence and conditional skewness seem rather precisely estimated. At the same time, the uniform confidence bands are wider, especially for the $\eta$ component.

Household Unobserved Heterogeneity. In Figure S15 of the Supplemental Material, we report the nonlinear persistence and conditional skewness of the $\eta$ component in model (20), which allows, in addition, for an additive household-specific effect. Compared to Figure 2, allowing for a household effect reduces persistence. Moreover, the nonlinear pattern is more pronounced than in the homogeneous case. Persistence is close to 1 for values of $\tau_{\text {init }}$ and $\tau_{\text {shock }}$ that are close to each other, but it is substantially lower when a large positive (respectively, negative) shock hits a low-earnings (resp., high-earnings) household.

Norwegian Population Register Data. The above results suggest that nonlinear persistence and conditional skewness are features of earnings processes in the PSID. In order to corroborate these findings using a different, larger data set, we estimated the earnings process using a balanced subsample of 2873 households from the 2000-2005 Norwegian administrative data, see Appendix C. The estimates are shown in Figures S16 to S19 of the Supplemental Material. Like the PSID, the Norwegian population register data present a similar pattern of conditional skewness. Moreover, the Norwegian data show similar nonlinear persistence in the persistent component $\eta$ as the PSID. At the same time, the dispersion of the transitory component $\varepsilon$ is much smaller in the Norwegian data, suggesting either the presence of large measurement error in the PSID or smaller true transitory innovations in Norway. In order to shed more light on the differences, it would be very interesting to also estimate our nonlinear model on a large administrative data set for the United States.

\subsection{Consumption}

We next turn to consumption. We estimated two main specifications: with and without household unobserved heterogeneity. Since, due to the small sample size in the PSID, allowing for unobserved heterogeneity is somewhat challenging on these data, here we focus on the results without unobserved heterogeneity. The results with unobserved heterogeneity are reported in the Supplemental Material.

Figure 5(a) shows estimates of the average derivative, with respect to $y_{i t}$, of the conditional mean of $c_{i t}$ given $y_{i t}, a_{i t}$, and $a g e_{i t}$. The function is evaluated at percentiles of log-assets and age $\left(\tau_{\text {assets }}\right.$ and $\tau_{\text {age }}$, respectively), and averaged over $y_{i t} .{ }^{35}$ The derivative

\footnotetext{
${ }^{35}$ We use tensor products of Hermite polynomials with degrees $(2,2,1)$ in the estimation of the consumption rule.
} 
(a) Response to earnings PSID data

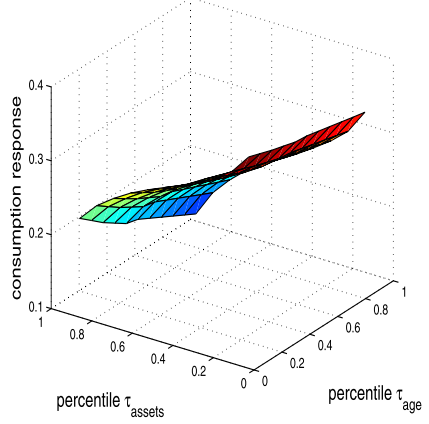

(b) Response to earnings Nonlinear model

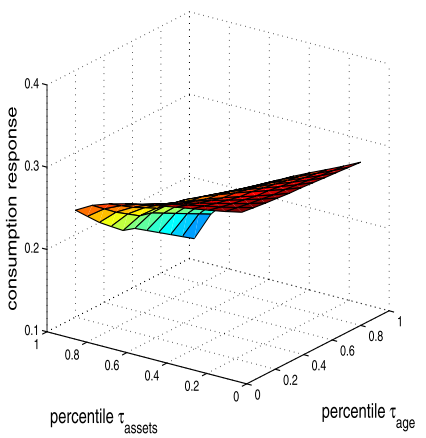

(c) Response to $\eta_{\text {it }}$ Nonlinear model

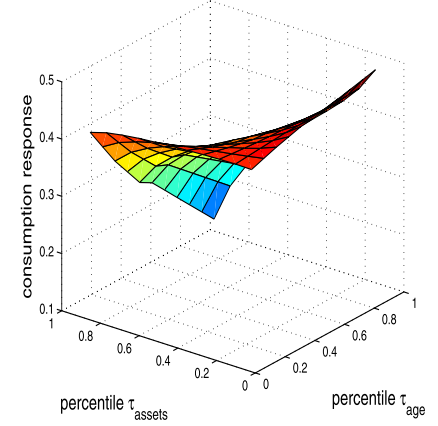

FIGURE 5.-Consumption responses to earnings shocks, by assets and age, model without household-specific unobserved heterogeneity. Note: Graphs (a) and (b) show estimates of the average derivative of the conditional mean of $c_{i t}$, with respect to $y_{i t}$, given $y_{i t}, a_{i t}$, and $a g e_{i t}$, evaluated at values of $a_{i t}$ and age $e_{i t}$ that correspond to their $\tau_{\text {assets }}$ and $\tau_{\text {age }}$ percentiles, and averaged over the values of $y_{i t}$. Graph (a) is based on the PSID data, and graph (b) is based on data simulated according to our nonlinear model with parameters set to their estimated values. Graph (c) shows estimates of the average consumption responses $\bar{\phi}_{t}(a)$ to variations in $\eta_{i t}$, evaluated at $\tau_{\text {assets }}$ and $\tau_{\text {age }}$.

effects lie between 0.2 and 0.3 . Moreover, the results indicate that consumption of older households, and of households with higher assets, is less correlated to variations in earnings. Figure 5(b) shows the same response surface based on simulated data from our full nonlinear model of earnings and consumption. The fit of the model, though not perfect, seems reasonable. In particular, the model reproduces the main pattern of correlation with age and assets. ${ }^{36,37}$

Figure 5(c) shows estimates of the average consumption response $\bar{\phi}_{t}(a)$ to variations in the persistent component of earnings. As described in Section 3, $1-\bar{\phi}_{t}(a)$ can be regarded as a measure of the degree of consumption insurability of shocks to the persistent earnings component, as a function of age and assets. On average, the estimated $\bar{\phi}_{t}(a)$ parameter lies between 0.3 and 0.4 , suggesting that more than half of pre-tax household earnings fluctuations is effectively insured. Moreover, variation in assets and age suggests the presence of an interaction effect. In particular, older households with high assets seem better insured against earnings fluctuations. ${ }^{38}$

In Figures S22 and S23 of the Supplemental Material, we report 95\% confidence bands for $\bar{\phi}_{t}(a)$ based on both parametric bootstrap and nonparametric bootstrap. The findings on insurability of shocks to the persistent earnings component seem quite precisely estimated. In Figure S24 of the Supplemental Material, we report estimates of the model with household unobserved heterogeneity in consumption; see (19). Estimated consump-

\footnotetext{
${ }^{36}$ In Figure S20 of the Supplemental Material, we show that the model fit to the density of log-consumption is also good.

${ }^{37}$ While the covariances between log-earnings and log-consumption residuals are well reproduced, the baseline model does not perform as well in fitting the dynamics of consumption, as it systematically underestimates the autocorrelations between log-consumption residuals. The specification with household unobserved heterogeneity improves the fit to consumption dynamics.

${ }^{38}$ Consumption responses to transitory shocks are shown in Figure S21 of the Supplemental Material.
} 
(a) Given $y_{i t}$

PSID data (b) Given $y_{i t}$

Nonlinear model (c) Given $\eta_{i t}$

Nonlinear model
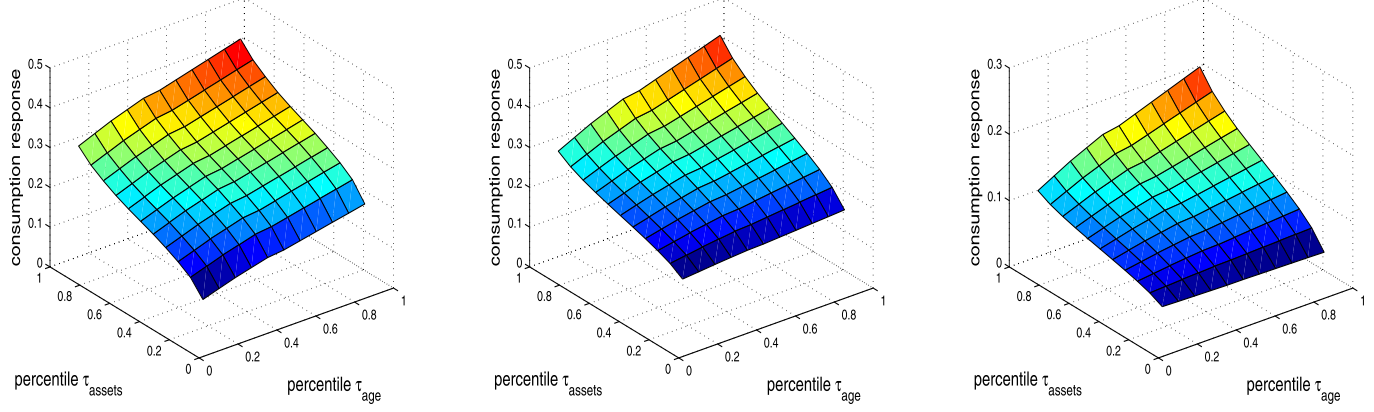

FIGURE 6.-Consumption responses to assets. Note: Estimates of the average derivative of the conditional mean of $c_{i t}$, with respect to $a_{i t}$, given $y_{i t}$ (respectively, given $\eta_{i t}$ and $\varepsilon_{i t}$ in graph (c)), $a_{i t}$, and $a g e_{i t}$, evaluated at values of $a_{i t}$ and age $e_{i t}$ that correspond to their $\tau_{\text {assets }}$ and $\tau_{\text {age }}$ percentiles, and averaged over the values of $y_{i t}$ (resp., over the values of $\eta_{i t}$ and $\varepsilon_{i t}$ in graph (c)). Model without unobserved heterogeneity.

tion responses are quite similar to the ones without unobserved heterogeneity, although the nonlinearity with respect to assets and age seems more pronounced.

Consumption Responses to Assets. In addition to consumption responses to earnings shocks, our nonlinear framework can be used to document derivative effects with respect to assets. Such quantities are often of great interest, for example when studying the implications of tax reforms. Graph (c) in Figure 6 shows estimated average derivatives, in a model without unobserved heterogeneity in consumption. ${ }^{39}$ The quantile polynomial specifications are the same as in Figure 5. We see that the responses range between 0.05 and 0.2 , and that the derivative effects seem to increase with age and assets.

\subsection{Simulating the Impact of Persistent Earnings Shocks}

In this last subsection, we simulate life-cycle earnings and consumption according to our nonlinear model, and show the evolution of earnings and consumption following a persistent earnings shock. In Figure 7, we report the difference between the age-specific medians of log-earnings of two types of households: households that are hit, at the same age 37 , by either a large negative shock to the persistent earnings component $\left(\tau_{\text {shock }}=0.10\right)$, or by a large positive shock $\left(\tau_{\text {shock }}=0.90\right)$, and households that are hit by a median shock $\tau=0.50$ to the persistent component. ${ }^{40}$ We report age-specific medians across 100,000 simulations of the model. At the start of the simulation (i.e., age 35), all households have the same persistent component indicated by the percentile $\tau_{\text {init }}$. With some abuse of terminology, we refer to the resulting earnings and consumption paths as "impulse responses." 41

\footnotetext{
${ }^{39}$ Graphs (a) and (b) in Figure 6 show that the model replicates well the empirical relationship between consumption and assets conditional on earnings and age. See Figure S25 of the Supplemental Material for the results in a model with unobserved heterogeneity.

${ }^{40}$ Note that such positive or negative shocks being "large" are relative statements, given that they correspond to ranks of different conditional distributions.

${ }^{41}$ See, for example, Gallant, Rossi, and Tauchen (1993) and Koop, Pesaran, and Potter (1996) for work on impulse response functions in nonlinear models.
} 
Nonlinear model

$\tau_{\text {init }}=.1$

(a) $\tau_{\text {shock }}=.1$

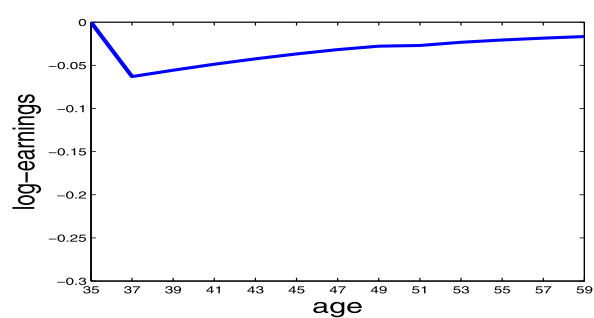

(b) $\tau_{\text {shock }}=.9$

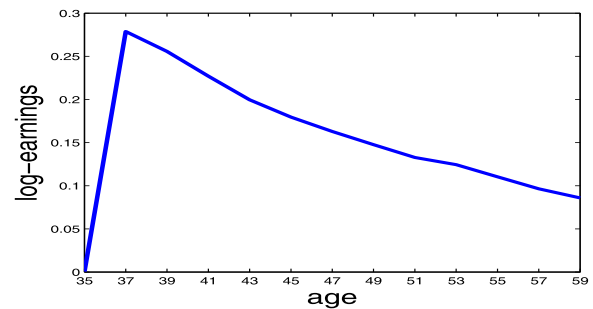

(c) $\tau_{\text {shock }}=.1$

$$
\tau_{\text {init }}=.5
$$

(d) $\tau_{\text {shock }}=.9$
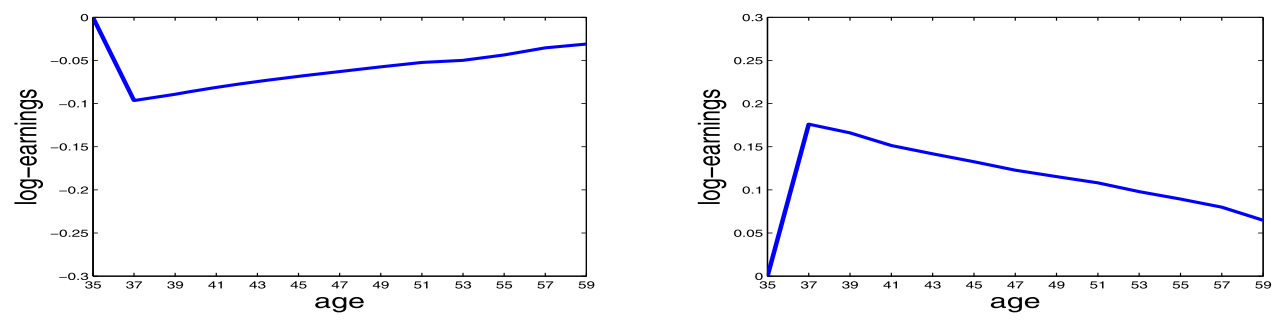

$$
\tau_{\text {init }}=.9
$$

(e) $\tau_{\text {shock }}=.1$

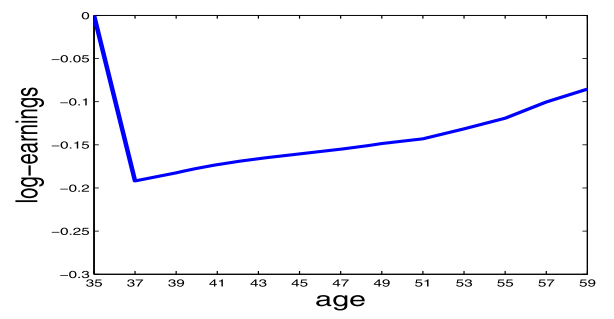

(f) $\tau_{\text {shock }}=.9$

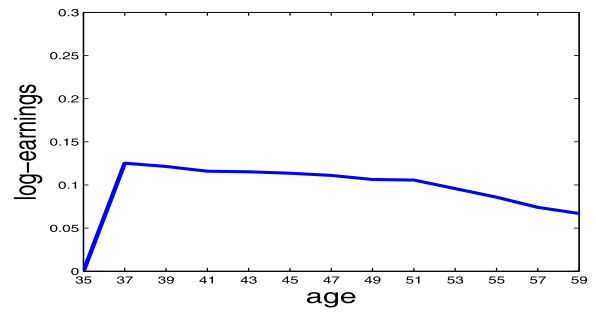

Canonical model

(g) $\tau_{\text {shock }}=.1$

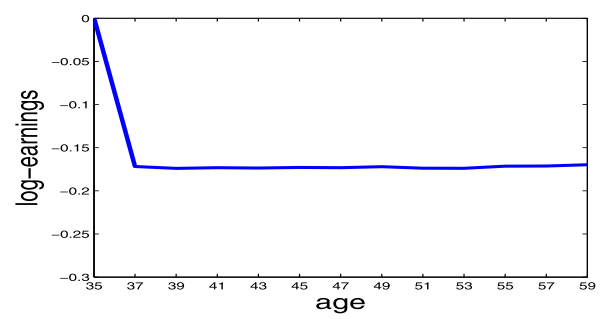

(h) $\tau_{\text {shock }}=.9$

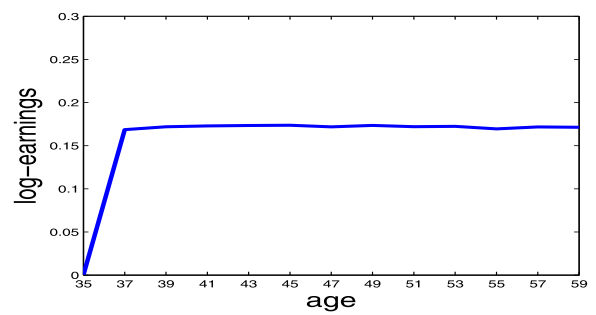

FIGURE 7.--Impulse responses, earnings. Note: Persistent component at percentile $\tau_{\text {init }}$ at age 35 . The graphs show the difference between a household hit by a shock $\tau_{\text {shock }}$ at age 37 , and a household hit by a 0.5 shock at the same age. Age-specific medians across 100,000 simulations. Graphs (a) to (f) correspond to the nonlinear model. Graphs (g) and (h) correspond to the canonical model (3) of earnings dynamics. 
Earnings responses reported in Figure 7 are consistent with the presence of interaction effects between the rank in the distribution of earnings component $\left(\tau_{\text {init }}\right)$ and the sign and size of the shock to the persistent component $\left(\tau_{\text {shock }}\right)$. While a large negative shock $\left(\tau_{\text {shock }}=0.10\right)$ is associated with a $7 \%$ drop in earnings for low-earnings households $\left(\tau_{\text {init }}=0.10\right)$, a similar shock is associated with a $19 \%$ drop for high-earnings households $\left(\tau_{\text {init }}=0.90\right)$. We also find interaction effects in the response to large positive shocks $\left(\tau_{\text {shock }}=0.90\right)$. This suggests the presence of asymmetries in the persistence of earnings histories, depending on the previous earnings history of the household and the size and magnitude of the shock. Moreover, the long-run impact of these shocks over the life-cycle also depends on the initial condition. For example, Figure 7(e) shows a very slow recovery from a negative earnings shock when starting from a high-earnings position, while graph (a) shows a quicker recovery.

These earnings impulse responses on impact are in line with the shape of the persistence function shown in Figure 2(d). In Figure 7, moving from panel (a) to panel (c) shows little difference in the impact of a shock, while moving to panel (e) shows a larger impact. This is consistent with the persistence function $\rho_{t}\left(\eta_{i, t-1}, \tau\right)$ being steeper in $\tau$ for high-income households who receive a large negative shock, compared to other households hit by such shock. Conversely, the earnings response is quite similar when moving between panels (f) and (d), while it is higher in panel (b), again in line with the shape of the persistence function.

In graphs (g) and (h) of Figure 7, we report results based on the "canonical model" of earnings dynamics where $\eta$ is a random walk; see equation (3). In this model, there are, by assumption, no interaction effects between income shocks and the ranks of households in the income distribution. The implications of the nonlinear earnings model thus differ markedly from those of standard linear models.

In Figure 8, we report the results of a similar exercise to Figure 7, but we now focus on consumption responses in a model without unobserved heterogeneity in consumption. In order to simulate consumption paths, one needs to take a stand on the rule of assets accumulation. We use the linear assets accumulation rule (7), with a constant (biennial) interest rate $r=3 \%$. In the simulation, we impose that $a_{i t} \geq 0$. We observed little sensitivity to varying the floor on assets.

We see that the nonlinearities observed in the earnings response matter for consumption, too. For example, while a large negative shock $\left(\tau_{\text {shock }}=0.10\right)$ is associated with a $2 \%$ drop in consumption for low-earnings households, it is associated with an $8 \%$ drop for high-earnings households. Conversely, a large positive shock is associated with a 5\% increase in consumption for high-earnings households, and with an $11 \%$ increase for lowearnings households. In Figures S26 to S29 of the Supplemental Material, we report bootstrap confidence bands for earnings and consumption responses, which suggest that the results are relatively precisely estimated. In addition, graphs $(\mathrm{g})$ and $(\mathrm{h})$ of Figure 8 report results based on the canonical earnings model with a linear log-consumption rule..$^{42}$ The fact that the canonical model assumes away the presence of interaction effects between income shocks and households' positions in the income distribution appears at odds with the data.

The results with unobserved heterogeneity in consumption are reported in Figure S30 of the Supplemental Material. They show smaller consumption responses to variations in earnings compared to the case without unobserved heterogeneity. For example, a large

\footnotetext{
${ }^{42}$ Specifically, $c_{i t}$ is modeled as a linear function of $\eta_{i t}, \varepsilon_{i t}$, and an independent additive error term i.i.d. over time. The model is estimated by equally-weighted minimum distance based on covariance restrictions.
} 
Nonlinear model

$$
\tau_{\text {init }}=.1
$$

(a) $\tau_{\text {shock }}=.1$

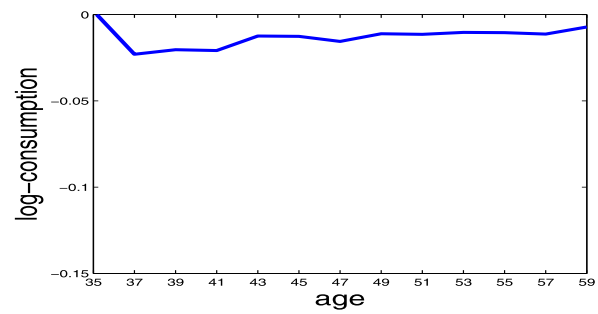

$$
\tau_{\text {init }}=.5
$$

(c) $\tau_{\text {shock }}=.1$
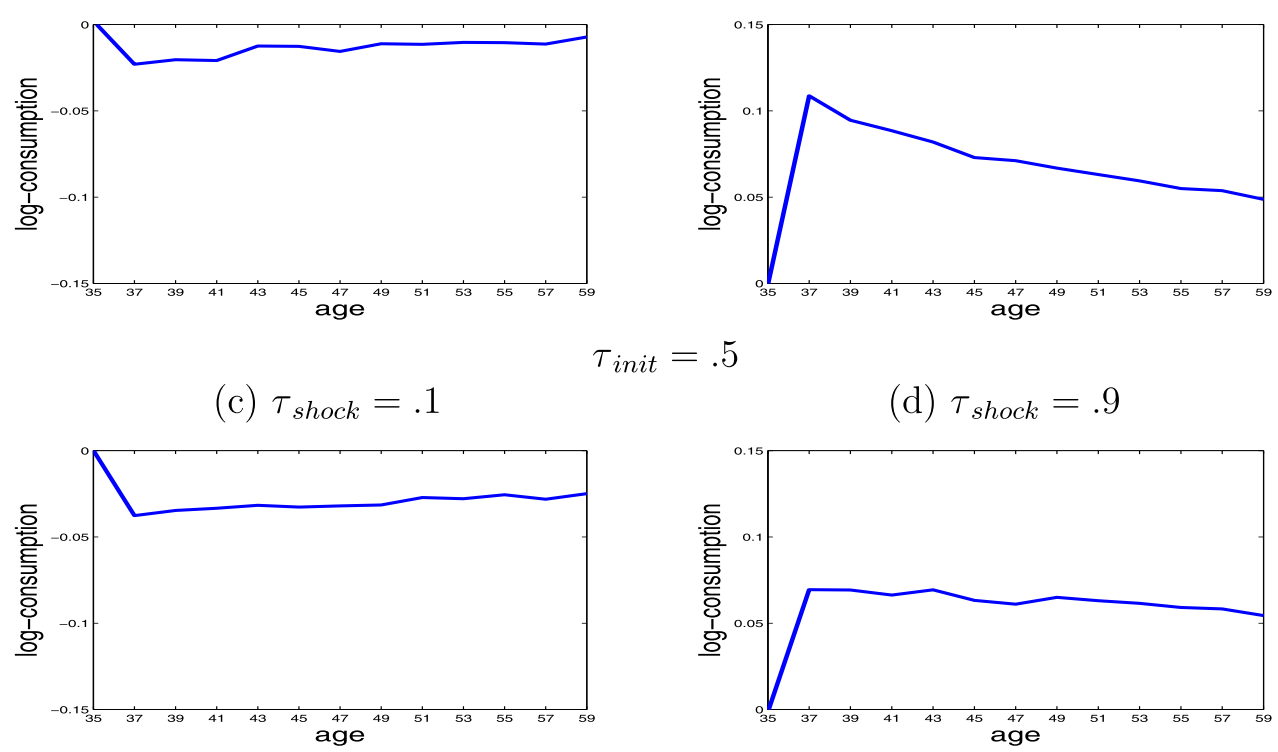

(b) $\tau_{\text {shock }}=.9$
(d) $\tau_{\text {shock }}=.9$
(1)

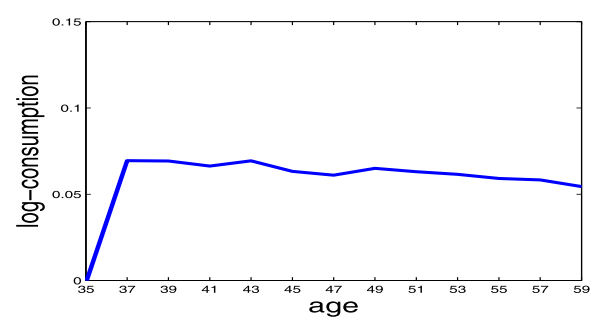

$\tau_{\text {init }}=.9$

(e) $\tau_{\text {shock }}=.1$

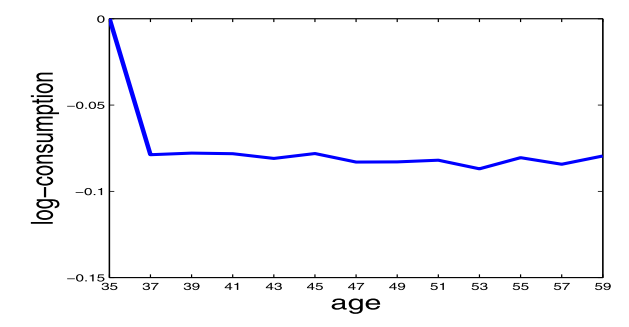

(f) $\tau_{\text {shock }}=.9$

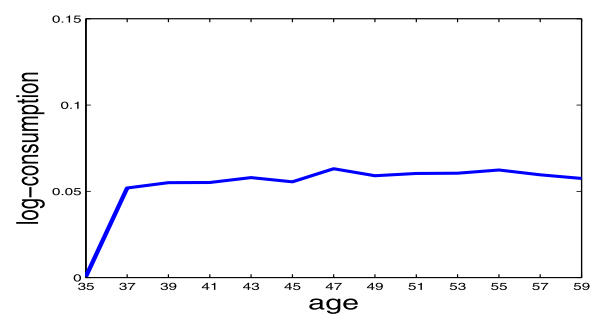

Canonical model

(g) $\tau_{\text {shock }}=.1$

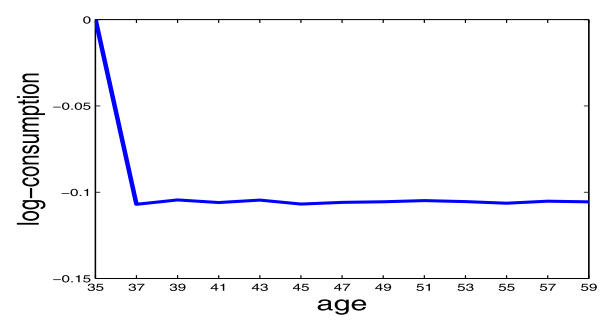

(h) $\tau_{\text {shock }}=.9$

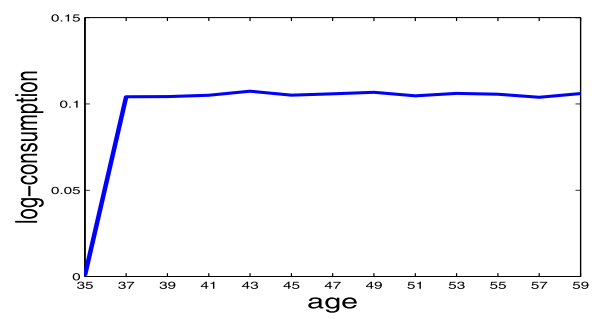

FIGURE 8.--Impulse responses, consumption. Note: See notes to Figure 7. Graphs (a) to (f) correspond to the nonlinear model. Graphs (g) and (h) correspond to the canonical model of earnings dynamics (3) and a linear consumption rule. Linear assets accumulation rule (7), $r=3 \% . a_{i t} \geq 0$. Model without household unobserved heterogeneity in consumption. 


\section{Earnings}

$$
\tau_{\text {init }}=.9, \tau_{\text {shock }}=.1
$$

(a) Young
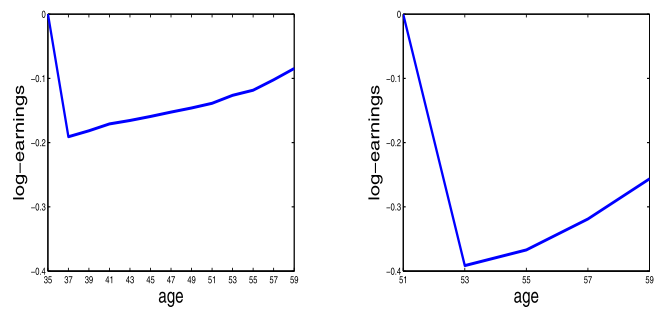

(c) Young

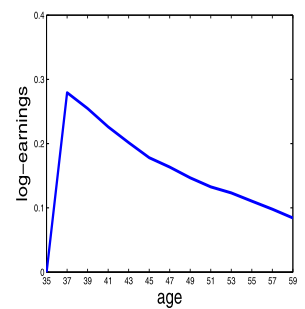

$$
\tau_{\text {init }}=.1, \tau_{\text {shock }}=.9
$$

(d) Old

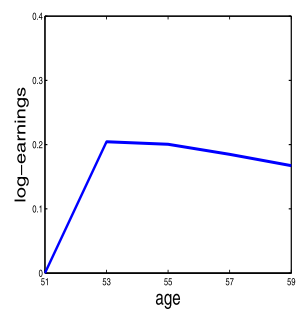

Consumption

(e) Young

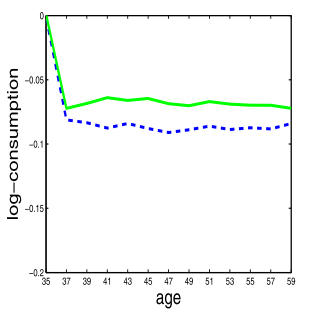

(f) Old

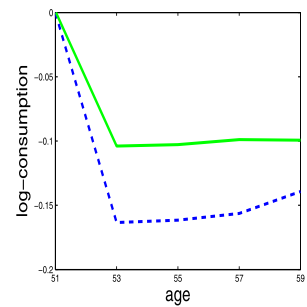

$\tau_{\text {init }}=.1, \tau_{\text {shock }}=.9$

(g) Young

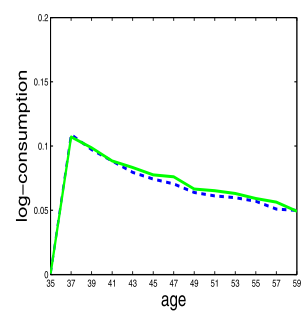

(h) Old

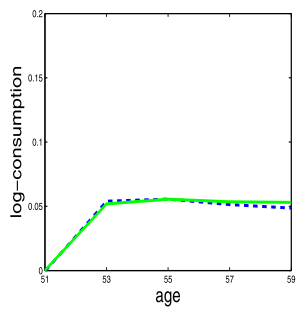

FIGURE 9.-Impulse responses by age and initial assets. Note: See notes to Figure 8. Initial assets at age 35 (for "young" households) or 51 (for "old" households) are at percentile 0.10 (dashed curves) and 0.90 (solid curves). Linear assets accumulation rule (7), $r=3 \% . a_{i t} \geq 0$. Model without household unobserved heterogeneity in consumption.

positive shock $\left(\tau_{\text {shock }}=0.90\right)$ is now associated with a $7 \%$ increase in consumption for low-earnings households. In addition, effects on consumption seem to revert more quickly towards the median in the model with heterogeneity. ${ }^{43}$

In Figure 9, we perform similar exercises, while varying the timing of shocks and the asset holdings that households possess. Graphs (a) to (d) suggest that a negative shock $\left(\tau_{\text {shock }}=0.10\right)$ for high-earnings households has a higher impact on earnings at later ages: the earnings drop is $40 \%$ when the shock hits at age 53 , compared to $20 \%$ when a similar shock hits at age 37 . The impact of a positive shock on low-earnings individuals seems to vary less with age. Graphs (e) to (h) in Figure 9 show the consumption responses in the model without heterogeneity. The results suggest that, while the presence of asset holdings does not seem to affect the insurability of positive earnings shocks, it does seem to attenuate the consumption response to negative shocks, particularly for households who are hit later in the life-cycle. Figure S33 of the Supplemental Material shows similar patterns when allowing for unobserved heterogeneity. ${ }^{4}$

\footnotetext{
${ }^{43}$ In Figures S31 and S32 of the Supplemental Material, we show the results from the nonlinear assets rule we have estimated; see (11). The results do not differ markedly compared to the baseline specification.

${ }^{44}$ The results for the estimated nonlinear assets rule reported in Figure S34 of the Supplemental Material show some differences compared to Figure 9, particularly for the responses to positive earnings shocks.
} 


\section{CONCLUSION}

In this paper, we have developed a nonlinear framework for modeling persistence that sheds new light on the nonlinear transmission of income shocks and the nature of consumption insurance. In this framework, household income is the sum of a first-order Markov persistent component and a transitory component. The consumption policy rule is an age-dependent, nonlinear function of assets, unobserved heterogeneity, persistent income, and transitory income. The model reveals asymmetric persistence patterns, where "unusual" earnings shocks are associated with a drop in persistence. It also leads to new empirical measures of partial insurance.

We provide conditions under which the model is nonparametrically identified, and we develop a tractable simulation-based sequential quantile regression method for estimation. These methods open the way to identify and estimate nonlinear models of earnings and consumption dynamics. They also provide new tools to assess the suitability of existing life-cycle models of consumption and savings, and potentially help guide the development of new structural models.

Our results suggest that nonlinear persistence and conditional skewness are important features of earnings processes. These features, which are present in both the PSID and the Norwegian population register data, are not easy to capture using existing models of earnings dynamics, motivating the use of new econometric methods to document distributional dynamics. Estimating models that allow for persistent and transitory components of income on a relatively homogeneous sample of households from the PSID, we find the presence of nonlinear persistence and conditional asymmetries in earnings.

The nonlinearities observed in the earnings responses are shown to impact consumption choices. For example, we found that while a large negative shock is associated with a relatively small drop in consumption for low-earnings households, it is associated with a sizable drop for high-earnings households. We also identified differences in persistence across different demographic groups. The results suggest that, while the presence of asset holdings seems not to affect the insurability of positive earnings shocks, it appears to attenuate consumption responses to negative shocks, particularly for households who are hit later in the life-cycle. Standard linear models, which assume away the presence of interaction effects between income shocks and the position in the income distribution, deliver qualitatively different predictions that appear at odds with the data.

A natural next step is to combine the framework introduced in this paper with more structural approaches. It is in fact easy to take our estimated Markovian earnings components to simulate or estimate fully-specified life-cycle models of consumption and savings. We provide an illustration of a simple life-cycle simulation model using the nonlinear dynamic quantile specification for earnings in Section S2 of the Supplemental Material. Moreover, the nonlinear model can be generalized to allow for other states and choices. For example, we could extend the analysis to incorporate intensive and extensive margins of labor supply, as in Low, Meghir, and Pistaferri (2010), and family labor supply, as in Blundell, Pistaferri, and Saporta-Eksten (2016).

Last, in this paper we have abstracted from the role of business-cycle fluctuations. In a recent paper on U.S. Social Security Data for 1978-2010, Guvenen, Ozcan, and Song (2014) found that the left-skewness of earnings shocks is countercyclical. In future work, it will be interesting to apply our framework to document distributional dynamics over the business-cycle. 


\section{APPENDIX A: IDENTIFICATION}

\section{A.1. Earnings Process}

In the following, all conditional and marginal densities are assumed to be bounded away from zero and infinity on their supports. With some abuse of notation, in the absence of ambiguity we use $f(a \mid b)$ as a generic notation for the conditional density $f_{A \mid B}(a \mid b)$, and for simplicity we omit the $i$ index in density arguments.

Operator Injectivity. The identification arguments below rely on the concept of operator injectivity, which we now formally define. A linear operator $\mathcal{L}$ is a linear mapping from a functional space $\mathcal{H}_{1}$ to another functional space $\mathcal{H}_{2} . \mathcal{L}$ is injective if the only solution $h \in \mathcal{H}_{1}$ to the equation $\mathcal{L} h=0$ is $h=0$.

One special case of operator injectivity ("deconvolution") obtains when $Y_{i 2}=Y_{i 1}+\epsilon_{i 1}$, with $Y_{i 1}$ independent of $\epsilon_{i 1}$, and $[\mathcal{L} h]\left(y_{2}\right)=\int h\left(y_{1}\right) f_{\epsilon_{1}}\left(y_{2}-y_{1}\right) d y_{1} . \mathcal{L}$ is then injective if the characteristic function of $\epsilon_{i 1}$ has no zeros on the real line. The normal and many other standard distributions satisfy this property. ${ }^{45}$ If the marginal distributions $f_{Y_{2}}$ and $f_{\epsilon_{1}}$ are known, injectivity implies that $h=f_{Y_{1}}$ is the only solution to the functional equation $\int h\left(y_{1}\right) f_{\epsilon_{1}}\left(y_{2}-y_{1}\right) d y_{1}=f_{Y_{2}}\left(y_{2}\right)$. In other words, $f_{Y_{1}}$ is identified from the knowledge of $f_{Y_{2}}$ and $f_{\epsilon_{1}}$.

Another, important special case of operator injectivity ("completeness") is obtained when $\mathcal{L}$ is the conditional expectation operator associated with the distribution of $\left(Y_{i 1} \mid Y_{i 2}\right)$, in which case $[\mathcal{L} h]\left(y_{2}\right)=\mathbb{E}\left[h\left(Y_{i 1}\right) \mid Y_{i 2}=y_{2}\right] . \mathcal{L}$ being injective is then equivalent to the distribution of $\left(Y_{i 1} \mid Y_{i 2}\right)$ being complete.

Building Block for Identification. To establish nonparametric identification of the earnings process, we rely on results from $\mathrm{Hu}$ and Schennach (2008) and Wilhelm (2015). In the context of a panel data model with measurement error, Wilhelm (2015) provided conditions under which the marginal distribution of $\varepsilon_{i 2}$ is identified, given three periods of observations $\left(y_{i 1}, y_{i 2}, y_{i 3}\right)$. We provide a brief summary of the identification argument used by Wilhelm in Section S4 of the Supplemental Material.

The key condition that underlies identification in this context is the fact that, in the earnings model with $T=3$, log-earnings $\left(y_{i 1}, y_{i 2}, y_{i 3}\right)$ are conditionally independent given $\eta_{i 2}{ }^{46}$ This "Hidden Markov" structure fits into the general setup considered in Hu and Schennach (2008). Hu (2015) provided a recent survey of applications of this line of work.

Identification of the Earnings Process. Returning to the earnings dynamics model (1)(2), let now $T \geq 3$. Suppose that the conditions in Wilhelm (2015) are satisfied on each of the three-year subpanels $t \in\{1,2,3\}$ to $t \in\{T-2, T-1, T\}$. It follows from Wilhelm's result that the marginal distributions of $\varepsilon_{i t}$ are identified for all $t \in\{2,3, \ldots, T-1\}$. By serial independence of the $\varepsilon$ 's, the joint distribution of $\left(\varepsilon_{i 2}, \varepsilon_{i 3}, \ldots, \varepsilon_{i, T-1}\right)$ is thus also identified.

Hence, if the characteristic functions of $\varepsilon_{i t}$ do not vanish on the real line, then by a deconvolution argument, the joint distribution of $\left(\eta_{i 2}, \eta_{i 3}, \ldots, \eta_{i, T-1}\right)$ is identified. As a result, all Markov transitions $f_{\eta_{t} \mid \eta_{t-1}}$ are identified for $t=3, \ldots, T-1$, and the marginal distribution of $\eta_{i 2}$ is identified as well (so we need $T \geq 4$ to identify at least one Markov transition). Moreover, it is easy to show that the conditional distributions of $\eta_{i 2} \mid y_{i 1}$ and $y_{i T} \mid \eta_{i, T-1}$ are identified. ${ }^{47}$

\footnotetext{
${ }^{45}$ Injectivity also holds if the zeros of the characteristic function of $\epsilon_{i 1}$ are isolated. See Evdokimov and White (2012).

${ }^{46}$ Indeed, $f\left(y_{1}, y_{2}, y_{3} \mid \eta_{2}\right)=f\left(y_{1} \mid \eta_{2}\right) f\left(y_{2} \mid \eta_{2}, y_{1}\right) f\left(y_{3} \mid \eta_{2}, y_{2}, y_{1}\right)=f\left(y_{1} \mid \eta_{2}\right) f\left(y_{2} \mid \eta_{2}\right) f\left(y_{3} \mid \eta_{2}\right)$.

${ }^{47}$ Indeed, we have $f_{y_{2} \mid y_{1}}\left(y_{2} \mid y_{1}\right)=\int f_{\varepsilon_{2}}\left(y_{2}-\eta_{2}\right) f_{\eta_{2} \mid y_{1}}\left(\eta_{2} \mid y_{1}\right) d \eta_{2}$. Hence, since the characteristic function of $\varepsilon_{i 2}$ is non-vanishing, $f_{\eta_{2} \mid y_{1}}\left(\cdot \mid y_{1}\right)$ is identified for given $y_{1}$. A similar argument shows that $f_{y_{T} \mid \eta_{T-1}}\left(y_{T} \mid \cdot\right)$ is identified for given $y_{T}$.
} 
Note that, in the case where $\varepsilon_{i 1}, \ldots, \varepsilon_{i T}$ have the same marginal distribution, then the distributions of the initial and terminal components $\varepsilon_{i 1}, \eta_{i 1}$, and $\varepsilon_{i T}, \eta_{i T}$ are also identified. However, the first- and last-period distributions are generally not identified in a fully non-stationary setting. In the empirical analysis, we impose time-stationarity restrictions, and pool different cohorts of households together in order to identify the distributions of $\eta$ 's and $\varepsilon$ 's at all ages. ${ }^{48}$

\section{A.2. Consumption Rule With Unobserved Heterogeneity}

We make the following assumption.

ASSUMPTION A.1:

(i) $u_{i, t+s}$ and $\varepsilon_{i, t+s}$, for all $s \geq 0$, are independent of $a_{i}^{t}, \eta_{i}^{t-1}, y_{i}^{t-1}$, and $\xi_{i} . \varepsilon_{i 1}$ is independent of $a_{i 1}, \eta_{i 1}$, and $\xi_{i}$.

(ii) $a_{i, t+1}$ is independent of $\left(a_{i}^{t-1}, c_{i}^{t-1}, y_{i}^{t-1}, \eta_{i}^{t-1}\right)$ conditional on $\left(a_{i t}, c_{i t}, y_{i t}, \eta_{i t}, \xi_{i}\right)$.

(iii) The taste shifter $\nu_{i t}$ in (19) is independent of $\eta_{i 1},\left(u_{i s}, \varepsilon_{i s}\right)$ for all $s, \nu_{i s}$ for all $s \neq t, a_{i}^{t}$, and $\xi_{i}$.

The identification strategy proceeds in two steps. First we have, by Assumption A.1(i) and (iii), for all $t \geq 1$,

$$
f\left(c^{t}, a^{t} \mid y\right)=\int f\left(c^{t}, a^{t} \mid \eta^{t}, y^{t}\right) f\left(\eta^{t} \mid y\right) d \eta^{t},
$$

or, equivalently,

$$
f\left(c^{t}, a^{t} \mid y\right)=\mathbb{E}\left[f\left(c^{t}, a^{t} \mid \eta_{i}^{t}, y_{i}^{t}\right) \mid y_{i}=y\right],
$$

where the expectation is taken for fixed $\left(c^{t}, a^{t}\right)$. Let $t=3 . f\left(c^{3}, a^{3} \mid \eta^{3}, y^{3}\right)$ is thus identified, provided the distribution of $\left(\eta_{i}^{3} \mid y_{i}\right)$ is boundedly complete in $\left(y_{i 4}, \ldots, y_{i T}\right)$. In particular, this argument requires that $T \geq 6$.

For the second step, we note that, by Assumption A.1,

$$
\begin{aligned}
f\left(c^{3}, a^{3} \mid \eta^{3}, y^{3}\right)= & \int f\left(a_{1}, c_{1}, a_{2} \mid \eta_{1}, y_{1}, \xi\right) f\left(c_{2}, a_{3} \mid a_{2}, \eta_{2}, y_{2}, \xi\right) \\
& \times f\left(c_{3} \mid a_{3}, \eta_{3}, y_{3}, \xi\right) f\left(\xi \mid \eta^{3}, y^{3}\right) d \xi
\end{aligned}
$$

For fixed $\left(a^{3}, \eta^{3}, y^{3}\right)$, equation (A.1) is formally analogous to the nonlinear instrumental variables setup of $\mathrm{Hu}$ and Schennach (2008). Hence the consumption rules, the asset evolution distributions, and the distribution of the latent heterogeneous component $\xi_{i}$, will all be nonparametrically identified under the conditions of $\mathrm{Hu}$ and Schennach's main theorem. These conditions include injectivity/completeness conditions analogous to the ones we have used in the baseline model, as well as a "scaling" condition. For example, in a consumption model that is additive in $\nu_{i t}$ (as in our empirical application), a possible

\footnotetext{
${ }^{48}$ Specifically, the above arguments allow to nonparametrically recover, for each cohort entering the sample at age $j$, the distributions of $\varepsilon$ at ages $j+2, j+4, j+6$, and $j+8$ (based on biennial data). In our data set, $j$ belongs to $\{25, \ldots, 50\}$. Pooling across cohorts, we obtain that the distributions of $\varepsilon$ are nonparametrically identified at all ages between 27 and 58 years. In turn, the joint distribution of $\eta$ 's is nonparametrically identified in this age range. Identification at ages 25, 26 and 59, 60 intuitively comes from parametric extrapolation using the quantile models.
} 
scaling condition (and the one we use) is that the mean of $c_{i 3}$, conditional on $\xi_{i}$ and some values of $\left(a_{i 3}, \eta_{i 3}, y_{i 3}\right)$, is increasing in $\xi_{i}$. In that case, identification is to be understood up to an increasing transformation of $\xi_{i} \cdot{ }^{49}$ Consumption rules and asset distributions for $t \geq 4$ can then be identified by relying on additional periods or, alternatively, under timestationarity assumptions by pooling information from different cohorts.

\section{APPENDIX B: ESTIMATION}

\section{B.1. Model's Restrictions}

Let $\rho_{\tau}(u)=u(\tau-\mathbf{1}\{u \leq 0\})$ denote the "check" function of quantile regression (Koenker and Bassett (1978)). Let also $\bar{\theta}$ denote the true value of $\theta$, and let

$$
f_{i}\left(\eta_{i}^{T} ; \bar{\theta}\right)=f\left(\eta_{i}^{T} \mid y_{i}^{T}, \operatorname{age}_{i}^{T} ; \bar{\theta}\right)
$$

denote the posterior density of $\eta_{i}^{T}=\left(\eta_{i 1}, \ldots, \eta_{i T}\right)$ given the earnings data. As the earnings model is fully specified, $f_{i}$ is a known function of $\bar{\theta}$.

We start by noting that, for all $\ell \in\{1, \ldots, L\}$,

$$
\begin{aligned}
\left(\bar{a}_{0 \ell}^{Q}, \ldots, \bar{a}_{K \ell}^{Q}\right)= & \underset{\left(a_{0 \ell}^{Q}, \ldots, a_{K \ell}^{Q}\right)}{\operatorname{argmin}} \sum_{t=2}^{T} \mathbb{E}\left[\int \rho_{\tau_{\ell}}\left(\eta_{i t}-\sum_{k=0}^{K} a_{k \ell}^{Q} \varphi_{k}\left(\eta_{i, t-1}, \text { age }_{i t}\right)\right)\right. \\
& \left.\times f_{i}\left(\eta_{i}^{T} ; \bar{\theta}\right) d \eta_{i}^{T}\right],
\end{aligned}
$$

where $\bar{a}_{k \ell}^{Q}$ denotes the true value of $a_{k \ell}^{Q}=a_{k}^{Q}\left(\tau_{\ell}\right)$, and the expectation is taken with respect to the distribution of $\left(y_{i}^{T}, a g e_{i}^{T}\right)$. To see that (B.1) holds, note that the objective function is smooth (due to the presence of the integrals) and convex (because of the "check" function). The first-order conditions of (B.1) are satisfied at true parameter values as, by (21), for all $k \in\{0, \ldots, K\}, \ell \in\{1, \ldots, L\}$, and $t \geq 2$,

$$
\mathbb{E}\left[\mathbf{1}\left\{\eta_{i t} \leq \sum_{k=0}^{K} \bar{a}_{k \ell}^{Q} \varphi_{k}\left(\eta_{i, t-1}, \text { age }_{i t}\right)\right\} \mid \eta_{i}^{t-1}, \text { age } e_{i}^{T}\right]=\tau_{\ell} .
$$

Likewise, we have, for all $\ell$,

$$
\begin{aligned}
\left(\bar{a}_{0 \ell}^{\varepsilon}, \ldots, \bar{a}_{K \ell}^{\varepsilon}\right)= & \underset{\left(a_{0 \ell}^{\varepsilon}, \ldots, a_{K \ell}^{\varepsilon}\right)}{\operatorname{argmin}} \sum_{t=1}^{T} \mathbb{E}\left[\int \rho_{\tau_{\ell}}\left(y_{i t}-\eta_{i t}-\sum_{k=0}^{K} a_{k \ell}^{\varepsilon} \varphi_{k}\left(\text { age }_{i t}\right)\right)\right. \\
& \left.\times f_{i}\left(\eta_{i}^{T} ; \bar{\theta}\right) d \eta_{i}^{T}\right]
\end{aligned}
$$

\footnotetext{
${ }^{49}$ Arellano and Bonhomme (2016) applied Hu and Schennach's (2008) results to a class of nonlinear panel data models.
} 
and, for all $\ell$,

$$
\left(\bar{a}_{0 \ell}^{\eta_{1}}, \ldots, \bar{a}_{K \ell}^{\eta_{1}}\right)=\underset{\left(a_{0 \ell}^{\eta_{1}}, \ldots, a_{K \ell}^{\eta_{1}}\right)}{\operatorname{argmin}} \mathbb{E}\left[\int \rho_{\tau_{\ell}}\left(\eta_{i 1}-\sum_{k=0}^{K} a_{k \ell}^{\eta_{1}} \varphi_{k}\left(\text { age }_{i 1}\right)\right) f_{i}\left(\eta_{i}^{T} ; \bar{\theta}\right) d \eta_{i}^{T}\right] .
$$

In addition to (B.1), (B.2), and (B.3), the model implies other restrictions on the tail parameters $\lambda$, which are given in the next subsection. All the restrictions depend on the posterior density $f_{i}$. Given the use of piecewise-linear interpolating splines, the joint likelihood function of $\left(\eta_{i}^{T}, y_{i}^{T} \mid a g e_{i}^{T} ; \bar{\theta}\right)$ is available in closed form, and we provide an explicit expression in the next subsection. In practice, this means that it is easy to simulate from $f_{i}$. We take advantage of this feature in our estimation algorithm.

Turning to consumption, we have

$$
\begin{aligned}
\left(\bar{\alpha}, \bar{b}_{1}^{g}, \ldots, \bar{b}_{K}^{g}\right)= & \underset{\left(\alpha, b_{1}^{g}, \ldots, b_{K}^{g}\right)}{\operatorname{argmin}} \sum_{t=1}^{T} \mathbb{E}\left[\int\left(c_{i t}-\alpha-\sum_{k=1}^{K} b_{k}^{g} \widetilde{\varphi}_{k}\left(a_{i t}, \eta_{i t}, y_{i t}-\eta_{i t}, \text { age }_{i t}\right)\right)^{2}\right. \\
& \left.\times g_{i}\left(\eta_{i}^{T} ; \bar{\theta}, \bar{\mu}\right) d \eta_{i}^{T}\right]
\end{aligned}
$$

where

$$
g_{i}\left(\eta_{i}^{T} ; \bar{\theta}, \bar{\mu}\right)=f\left(\eta_{i}^{T} \mid c_{i}^{T}, a_{i}^{T}, y_{i}^{T}, a g e_{i}^{T} ; \bar{\theta}, \bar{\mu}\right)
$$

denotes the posterior density of $\left(\eta_{i 1}, \ldots, \eta_{i T}\right)$ given the earnings, consumption, and asset data.

Moreover, the variance of taste shifters satisfies

$$
\begin{aligned}
\bar{\sigma}^{2}= & \frac{1}{T} \sum_{t=1}^{T} \mathbb{E}\left[\int\left(c_{i t}-\bar{\alpha}-\sum_{k=1}^{K} \bar{b}_{k}^{g} \widetilde{\varphi}_{k}\left(a_{i t}, \eta_{i t}, y_{i t}-\eta_{i t}, a g e_{i t}\right)\right)^{2}\right. \\
& \left.\times g_{i}\left(\eta_{i}^{T} ; \bar{\theta}, \bar{\mu}\right) d \eta_{i}^{T}\right] .
\end{aligned}
$$

Likewise, for assets we have

$$
\begin{aligned}
\left(\bar{\alpha}^{h}, \bar{b}_{1}^{h}, \ldots, \bar{b}_{K}^{h}\right)= & \underset{\left(\alpha^{h}, b_{1}^{h}, \ldots, b_{K}^{h}\right)}{\operatorname{argmin}} \sum_{t=2}^{T} \mathbb{E}\left[\int \left(a_{i t}-\alpha^{h}\right.\right. \\
& \left.\left.-\sum_{k=1}^{K} b_{k}^{h} \widetilde{\varphi}_{k}\left(a_{i, t-1}, c_{i, t-1}, y_{i, t-1}, \eta_{i, t-1}, \text { age }_{i t}\right)\right)^{2} g_{i}\left(\eta_{i}^{T} ; \bar{\theta}, \bar{\mu}\right) d \eta_{i}^{T}\right],
\end{aligned}
$$

with a similar expression for the variance of $b_{0}^{h}\left(v_{i t}\right)$ as in (B.4).

Last, we have, for all $\ell$,

$$
\left(\bar{b}_{0 \ell}^{a}, \ldots, \bar{b}_{K \ell}^{a}\right)=\underset{\left(b_{0 \ell}^{a}, \ldots, b_{K \ell}^{a}\right)}{\operatorname{argmin}} \mathbb{E}\left[\int \rho_{\tau_{\ell}}\left(a_{i 1}-\sum_{k=0}^{K} b_{k \ell}^{a} \widetilde{\varphi}_{k}\left(\eta_{i 1}, a^{2} e_{i 1}\right)\right) g_{i}\left(\eta_{i}^{T} ; \bar{\theta}, \bar{\mu}\right) d \eta_{i}^{T}\right],
$$

with additional restrictions characterizing tail parameters given in the next subsection. 


\section{B.2. Estimation Algorithm}

Additional Model Restrictions. The tail parameters $\lambda$ satisfy simple moment restrictions. For example, we have

$$
\bar{\lambda}_{-}^{Q}=-\frac{\sum_{t=2}^{T} \mathbb{E}\left[\int \mathbf{1}\left\{\eta_{i t} \leq \sum_{k=0}^{K} \bar{a}_{k 1}^{Q} \varphi_{k}\left(\eta_{i, t-1}, a g e_{i t}\right)\right\} f_{i}\left(\eta_{i}^{T} ; \bar{\theta}\right) d \eta_{i}^{T}\right]}{\sum_{t=2}^{T} \mathbb{E}\left[\int\left(\eta_{i t}-\sum_{k=0}^{K} \bar{a}_{k 1}^{Q} \varphi_{k}\left(\eta_{i, t-1}, \text { age }_{i t}\right)\right) \mathbf{1}\left\{\eta_{i t} \leq \sum_{k=0}^{K} \bar{a}_{k 1}^{Q} \varphi_{k}\left(\eta_{i, t-1}, \text { age }_{i t}\right)\right\} f_{i}\left(\eta_{i}^{T} ; \bar{\theta}\right) d \eta_{i}^{T}\right]},
$$

and

$$
\bar{\lambda}_{+}^{Q}=\frac{\sum_{t=2}^{T} \mathbb{E}\left[\int \mathbf{1}\left\{\eta_{i t} \geq \sum_{k=0}^{K} \bar{a}_{k L}^{Q} \varphi_{k}\left(\eta_{i, t-1}, \text { age }_{i t}\right)\right\} f_{i}\left(\eta_{i}^{T} ; \bar{\theta}\right) d \eta_{i}^{T}\right]}{\sum_{t=2}^{T} \mathbb{E}\left[\int\left(\eta_{i t}-\sum_{k=0}^{K} \bar{a}_{k L}^{Q} \varphi_{k}\left(\eta_{i, t-1}, a g e_{i t}\right)\right) \mathbf{1}\left\{\eta_{i t} \geq \sum_{k=0}^{K} \bar{a}_{k L}^{Q} \varphi_{k}\left(\eta_{i, t-1}, \text { age }_{i t}\right)\right\} f_{i}\left(\eta_{i}^{T} ; \bar{\theta}\right) d \eta_{i}^{T}\right]},
$$

with similar equations for the other tail parameters.

Likelihood Function. The likelihood function is (omitting the conditioning on age for conciseness)

$$
\begin{aligned}
f\left(y_{i}^{T}, c_{i}^{T}, a_{i}^{T}, \eta_{i}^{T} ; \theta, \mu\right)= & \prod_{t=1}^{T} f\left(y_{i t} \mid \eta_{i t} ; \theta\right) \prod_{t=1}^{T} f\left(c_{i t} \mid a_{i t}, \eta_{i t}, y_{i t} ; \mu\right) \\
& \times \prod_{t=2}^{T} f\left(a_{i t} \mid a_{i, t-1}, y_{i, t-1}, c_{i, t-1}, \eta_{i, t-1} ; \mu\right) \\
& \times \prod_{t=2}^{T} f\left(\eta_{i t} \mid \eta_{i, t-1} ; \theta\right) f\left(a_{i 1} \mid \eta_{i 1} ; \mu\right) f\left(\eta_{i 1} ; \theta\right) .
\end{aligned}
$$

The likelihood function is fully specified and available in closed form. For example, we have

$$
\begin{aligned}
f\left(y_{i t} \mid \eta_{i t} ; \theta\right)= & \mathbf{1}\left\{y_{i t}-\eta_{i t}<A_{i t}^{\varepsilon}(1)\right\} \tau_{1} \lambda_{-}^{\varepsilon} \exp \left[\lambda_{-}^{\varepsilon}\left(y_{i t}-\eta_{i t}-A_{i t}^{\varepsilon}(1)\right)\right] \\
& +\sum_{\ell=1}^{L-1} \mathbf{1}\left\{A_{i t}^{\varepsilon}(\ell) \leq y_{i t}-\eta_{i t}<A_{i t}^{\varepsilon}(\ell+1)\right\} \frac{\tau_{\ell+1}-\tau_{\ell}}{A_{i t}^{\varepsilon}(\ell+1)-A_{i t}^{\varepsilon}(\ell)} \\
& +\mathbf{1}\left\{A_{i t}^{\varepsilon}(L) \leq y_{i t}-\eta_{i t}\right\}\left(1-\tau_{L}\right) \lambda_{+}^{\varepsilon} \exp \left[-\lambda_{+}^{\varepsilon}\left(y_{i t}-\eta_{i t}-A_{i t}^{\varepsilon}(L)\right)\right],
\end{aligned}
$$

where $A_{i t}^{\varepsilon}(\ell) \equiv \sum_{k=0}^{K} a_{k \ell}^{\varepsilon} \varphi_{k}\left(a g e_{i t}\right)$ for all $(i, t, \ell)$. Note that the likelihood function is nonnegative by construction. In particular, drawing from the posterior density of $\eta$ automati- 
cally produces rearrangement of the various quantile curves (Chernozhukov, FernándezVal, and Galichon (2010)).

Estimation Algorithm: Earnings. Start with $\widehat{\theta}^{(0)}$. Iterate on $s=0,1,2, \ldots$ the two following steps:

Stochastic E-step: Draw $M$ values $\eta_{i}^{(m)}=\left(\eta_{i 1}^{(m)}, \ldots, \eta_{i T}^{(m)}\right)$ from

$$
f\left(\eta_{i}^{T} \mid y_{i}^{T} ; \widehat{\theta}^{(s)}\right) \propto \prod_{t=1}^{T} f\left(y_{i t} \mid \eta_{i t} ; \widehat{\theta}^{(s)}\right) f\left(\eta_{i 1} ; \widehat{\theta}^{(s)}\right) \prod_{t=2}^{T} f\left(\eta_{i t} \mid \eta_{i, t-1} ; \widehat{\theta}^{(s)}\right)
$$

where $a \propto b$ means that $a$ and $b$ are equal up to a proportionality factor independent of $\eta$.

M-step: Compute, ${ }^{50}$ for $\ell=1, \ldots, L$,

$$
\begin{aligned}
\left(\widehat{a}_{0 \ell}^{Q,(s+1)}, \ldots, \widehat{a}_{K \ell}^{Q,(s+1)}\right) & =\underset{\left(a_{0 \ell}^{Q}, \ldots, a_{K \ell}^{Q}\right)}{\operatorname{argmin}} \sum_{i=1}^{N} \sum_{t=2}^{T} \sum_{m=1}^{M} \rho_{\tau_{\ell}}\left(\eta_{i t}^{(m)}-\sum_{k=0}^{K} a_{k \ell}^{Q} \varphi_{k}\left(\eta_{i, t-1}^{(m)}, \text { age }_{i t}\right)\right), \\
\left(\widehat{a}_{0 \ell}^{\varepsilon,(s+1)}, \ldots, \widehat{a}_{K \ell}^{\varepsilon,(s+1)}\right) & =\underset{\left(a_{0 \ell}^{\varepsilon}, \ldots, a_{K \ell}^{\varepsilon}\right)}{\operatorname{argmin}} \sum_{i=1}^{N} \sum_{t=1}^{T} \sum_{m=1}^{M} \rho_{\tau_{\ell}}\left(y_{i t}-\eta_{i t}^{(m)}-\sum_{k=0}^{K} a_{k \ell}^{\varepsilon} \varphi_{k}\left(\text { age }_{i t}\right)\right), \\
\left(\widehat{a}_{0 \ell}^{\eta_{1},(s+1)}, \ldots, \widehat{a}_{K \ell}^{\eta_{1},(s+1)}\right) & =\underset{\left(a_{0 \ell}^{\eta_{1}}, \ldots, a_{K \ell}^{\eta_{1}}\right)}{\operatorname{argmin}} \sum_{i=1}^{N} \sum_{m=1}^{M} \rho_{\tau_{\ell}}\left(\eta_{i 1}^{(m)}-\sum_{k=0}^{K} a_{k \ell}^{\eta_{1}} \varphi_{k}\left(\operatorname{age}_{i 1}\right)\right),
\end{aligned}
$$

and compute

$$
\widehat{\lambda}_{-}^{Q,(s+1)}=-\frac{\sum_{i=1}^{N} \sum_{t=2}^{T} \sum_{m=1}^{M} \mathbf{1}\left\{\boldsymbol{\eta}_{i t}^{(m)} \leq \widehat{A}_{i t m}^{Q,(s+1)}\right\}}{\sum_{i=1}^{N} \sum_{t=2}^{T} \sum_{m=1}^{M}\left(\eta_{i t}^{(m)}-\widehat{A}_{i t m}^{Q,(s+1)}\right) \mathbf{1}\left\{\eta_{i t}^{(m)} \leq \widehat{A}_{i t m}^{Q,(s+1)}\right\}},
$$

where

$$
\widehat{A}_{i t m}^{Q,(s+1)} \equiv \sum_{k=0}^{K} \widehat{a}_{k 1}^{Q,(s+1)} \varphi_{k}\left(\eta_{i, t-1}^{(m)}, a g e_{i t}\right),
$$

with similar updating rules for $\widehat{\lambda}_{+}^{Q,(s+1)}, \widehat{\lambda}_{-}^{\varepsilon,(s+1)}, \widehat{\lambda}_{+}^{\varepsilon,(s+1)}, \widehat{\lambda}_{-}^{\eta_{1},(s+1)}$, and $\widehat{\lambda}_{+}^{\eta_{1},(s+1)}$.

In practice, we start the algorithm with different choices for $\widehat{\theta}^{(0)}$. For example, for the initial values of the quantile parameters in $\eta_{i t}$, we run quantile regressions of log-earnings on lagged log-earnings and age. We proceed similarly to set other starting parameter values. We experimented with a number of other choices, and selected the parameter values corresponding to the highest average log-likelihood over iterations. We observed some effect of starting values on estimates of tail parameters, although most results were stable.

\footnotetext{
${ }^{50}$ In practice, we used gradient descent algorithms in all M-step computations. This provided the fastest alternative in our repeated estimation, without any noticeable loss in accuracy.
} 
Estimation Algorithm: Consumption. Similar to the earnings case. One difference is that in the stochastic E-step we draw $\eta_{i}^{(m)}$ from

$$
\begin{aligned}
f\left(\eta_{i}^{T} \mid y_{i}^{T}, c_{i}^{T}, a_{i}^{T} ; \widehat{\theta}, \widehat{\mu}^{(s)}\right) \propto & \prod_{t=1}^{T} f\left(y_{i t} \mid \eta_{i t} ; \widehat{\theta}\right) f\left(\eta_{i 1} ; \widehat{\theta}\right) \prod_{t=2}^{T} f\left(\eta_{i t} \mid \eta_{i, t-1} ; \widehat{\theta}\right) \\
& \times f\left(a_{i 1} \mid \eta_{i 1} ; \widehat{\mu}^{(s)}\right) \prod_{t=2}^{T} f\left(a_{i t} \mid a_{i, t-1}, c_{i, t-1}, y_{i, t-1}, \eta_{i, t-1} ; \widehat{\mu}^{(s)}\right) \\
& \times \prod_{t=1}^{T} f\left(c_{i t} \mid a_{i t}, \eta_{i t}, y_{i t} ; \widehat{\mu}^{(s)}\right) .
\end{aligned}
$$

\section{APPENDIX C: DATA APPENDIX}

\section{C.1. PSID Data}

We use the 1999-2009 Panel Study of Income Dynamics (PSID) to estimate the model. The PSID started in 1968 collecting information on a sample of roughly 5000 households. Of these, about 3000 were representative of the U.S. population as a whole (the core sample), and about 2000 were low-income families (the Census Bureau's SEO sample). Thereafter, both the original families and their split-offs (children of the original family forming a family of their own) have been followed. The PSID data were collected annually until 1996 and biennially starting in 1997. A great advantage of PSID after 1999 is that, in addition to income data and demographics, it collects data about detailed asset holdings and consumption expenditures in each wave. To the best of our knowledge, this makes the PSID the only representative large-scale U.S. panel to include income, hours, consumption, and assets data. Since we need both consumption and assets data, we focus on the 1999-2009 sample period.

We focus on non-SEO households with participating and married male household heads aged between 25 and 60, and with non-missing information on key demographics (age, education, and state of residence). To reduce the influence of measurement error, we also drop observations with extremely high asset values (20 millions or more), as well as observations with total transfers more than twice the size of total household earnings. When calculating the relevant consumption, hourly wage, and earnings moments, we do not use data displaying extreme "jumps" from one year to the next (most likely due to measurement error). Furthermore, we do not use earnings and wage data when the implied hourly wage is below one-half the state minimum wage. See Blundell, Pistaferri, and Saporta-Eksten (2016) for further details of the sample selection.

\section{C.2. Norwegian Population Register Data}

Sample Selection. The Norwegian results are provided as part of the Blundell, Graber, and Mogstad project on "Labour Income Dynamics and the Insurance from Taxes, Transfers and the Family'; see Blundell, Graber, and Mogstad (2015) for details. For Figure 1, we use Norwegian register data provided under that project for the years 2005 and 2006 only.

We select a balanced panel of households where the male head is Norwegian, resident in Norway. We restrict the sample to include male, non-immigrant residents between the age 30 and 60 and their spouse (if they have one), with non-missing information on all 
TABLE C.I

DESCRIPTIVE STATISTICS

\begin{tabular}{|c|c|c|c|c|c|c|}
\hline & 1999 & 2001 & 2003 & 2005 & 2007 & 2009 \\
\hline & \multicolumn{6}{|c|}{ Earnings } \\
\hline Mean & 87,120 & 93,777 & 96,289 & 98,475 & 103,442 & 102,893 \\
\hline $10 \%$ & 34,863 & 37,532 & 36,278 & 35,005 & 35,533 & 31,992 \\
\hline $25 \%$ & 50,709 & 53,000 & 52,975 & 54,696 & 53,813 & 52,451 \\
\hline $50 \%$ & 73,423 & 77,000 & 76,576 & 78,944 & 80,292 & 79,181 \\
\hline $75 \%$ & 102,211 & 106,000 & 105,292 & 109,391 & 113,604 & 112,607 \\
\hline \multirow[t]{2}{*}{$90 \%$} & 145,789 & 152,000 & 150,280 & 154,971 & 171,688 & 163,879 \\
\hline & \multicolumn{6}{|c|}{ Consumption } \\
\hline Mean & 30,761 & 34,784 & 37,553 & 43,199 & 44,511 & 40,598 \\
\hline $10 \%$ & 15,804 & 17,477 & 18,026 & 20,365 & 21,634 & 20,008 \\
\hline $25 \%$ & 20,263 & 21,786 & 22,834 & 26,322 & 28,341 & 26,167 \\
\hline $50 \%$ & 26,864 & 29,366 & 31,924 & 37,381 & 38,704 & 34,570 \\
\hline $75 \%$ & 36,887 & 41,030 & 45,071 & 51,529 & 53,239 & 47,300 \\
\hline \multirow[t]{2}{*}{$90 \%$} & 48,977 & 53,870 & 62,864 & 73,338 & 73,715 & 67,012 \\
\hline & \multicolumn{6}{|c|}{ Net worth } \\
\hline Mean & 224,127 & 283,539 & 311,664 & 387,830 & 447,323 & 406,290 \\
\hline $10 \%$ & 19,016 & 26,100 & 28,494 & 38,287 & 41,854 & 33,592 \\
\hline $25 \%$ & 48,095 & 59,600 & 69,397 & 83,137 & 101,005 & 85,179 \\
\hline $50 \%$ & 114,096 & 137,500 & 159,230 & 191,663 & 217,599 & 188,354 \\
\hline $75 \%$ & 248,000 & 301,750 & 345,549 & 413,955 & 489,224 & 384,625 \\
\hline $90 \%$ & 535,827 & 586,000 & 654,437 & 830,462 & 939,583 & 867,786 \\
\hline
\end{tabular}

a Balanced subsample from PSID, 1999-2009. $N=792, T=6$. In 2001 dollars.

key demographic variables. We choose a balanced panel of continuously married males, where household disposable income (i.e., pooled labor income after tax and transfers of the spouses) is above the threshold of substantial gainful activity (one basic amount, 14,000 USD in 2014) and where the total income from self-employment is below one basic amount.

The residual income measure is obtained by regressing the log of household disposable income on a set of demographics including cohort interacted with education category of both spouses, dummies for children and region. Our measure of household disposable income pools the individual disposable income of the spouses (if the male has a spouse). In each year, we regress the log of household disposable income on dummies for region, marital status, number of children, education, and a fourth-order polynomial in age and the interaction of the latter two to obtain the residual income. To produce Figure 1, we use an equidistant grid of 11 quantiles and a third-order Hermite polynomial.

\section{REFERENCES}

ABOWD, J., AND D. CARD (1989): “On the Covariance Structure of Earnings and Hours Changes," Econometrica, 57 (2), 411-445. [694]

ALAN, S. (2012): “Do Disaster Expectations Explain Household Portfolios?” Quantitative Economics, 3, 1-28. [704]

Alan, S., M. Browning, AND M. EjRnaes (2014): "Income and Consumption: A Micro Semi-Structural Analysis With Pervasive Heterogeneity," Report, University of Essex. [694]

ANDREwS, D. (2011): "Examples of $L^{2}$-Complete and Boundedly-Complete Distributions," Unpublished Manuscript. [707] 
Arellano, M., S. Bonhomme (2016): “Nonlinear Panel Data Estimation via Quantile Regressions,” Econometrics Journal, 19, C61-C94. [696,709-711,726]

AREllano, M., R. BLundell, AND S. BonhOmme (2017): "Supplement to 'Earnings and Consumption Dynamics: A Nonlinear Panel Data Framework'," Econometrica Supplemental Material, 85, http://dx.doi.org/10. 3982/ECTA13795. [697]

BARRo, R. (2006): "Rare Disasters and Asset Markets in the Twentieth Century," Quarterly Journal of Economics, 121 (3), 823-866. [695]

Belloni, A., V. Chernozhukov, D. Chetverikov, And I. Fernández-VAl (2016): "Conditional Quantile Processes Based on Series or Many Regressors," Unpublished Manuscript. [711]

BLundell, R. (2014): "Income Dynamics and Life-Cycle Inequality: Mechanisms and Controversies," Economic Journal, 124 (2), 289-318. [693]

Blundell, R., X. Chen, AND D. KRISTENSEN (2007): "Semi-Nonparametric IV Estimation of ShapeInvariant Engel Curves," Econometrica, 75 (6), 1613-1669. [705]

Blundell, R., M. GRABER, AND M. Mogstad (2015): "Labor Income Dynamics and the Insurance From Taxes, Transfers, and the Family," Journal of Public Economics, 127, 58-73. [730]

BLundell, R., L. Pistaferri, AND I. PRESTON (2008): "Consumption Inequality and Partial Insurance," American Economic Review, 98 (5), 1887-1921. [694,696,707]

BLUNDELl, R., L. PISTAFERRI, AND I. SAPORTA-EKSTEN (2016): "Consumption Smoothing and Family Labor Supply," American Economic Review, 106 (2), 387-435. [696,712,723,730]

Bonhomme, S., AND J. M. RoBIN (2010): "Generalized Nonparametric Deconvolution With an Application to Earnings Dynamics," Review of Economic Studies, 77 (2), 491-533. [697,714]

Botosaru, I., AND Y. SASAKI (2015): "Nonparametric Heteroskedasticity in Persistent Panel Processes: An Application to Earnings Dynamics," Unpublished Manuscript. [695]

Bound, J., C. BRown, AND N. MATHIOWETZ (2001): "Measurement Error in Survey Data," in Handbook of Econometrics, Vol. 5, ed. by J. Heckman and E. Leamer. Amsterdam: North-Holland. [698]

Browning, M., M. EJRnAes, AND J. Alvarez (2010): "Modelling Income Processes With Lots of Heterogeneity," Review of Economic Studies, 77 (4), 1353-1381. [697]

CASTAÑeda, A., J. DíAZ-GimÉnez, And J. V. Ríos-Rull (2003): "Accounting for the U.S. Earnings and Wealth Inequality," Journal of Political Economy, 111 (4), 818-857. [695]

CeleuX, G., AND J. Diebolt (1993): "Asymptotic Properties of a Stochastic EM Algorithm for Estimating Mixing Proportions," Communications in Statistics. Stochastic Models, 9, 599-613. [710]

Chernozhukov, V., I. FernándeZ-VAL, AND A. Galichon (2010): "Quantile and Probability Curves Without Crossing," Econometrica, 78 (3), 1093-1125. [729]

Constandinides, G., AND A. Gosh (2017): "Asset Pricing With Countercyclical Household Consumption Risk," Journal of Finance, 72 (1), 415-460. [695]

DEATON, A., AND C. PAXSON (1994): "Intertemporal Choice and Inequality," Journal of Political Economy, 102 (3), 437-467. [693]

DempSTER, A. P., N. M. LAIRD, AND D. B. Rubin (1977): "Maximum Likelihood From Incomplete Data via the EM Algorithm," Journal of the Royal Statistical Society, Series B, 39, 1-38. [710]

D'Haultfoeuille, X. (2011): "On the Completeness Condition for Nonparametric Instrumental Problems," Econometric Theory, 27, 460-471. [707]

EVANS, M., AND P. WACHTEL (1993): "Inflation Regimes and the Sources of Inflation Uncertainty," Journal of Money, Credit, and Banking, 25, 475-511. [697]

EvDOKIMOV, K., AND H. WHITE (2012): “Some Extensions of a Lemma of Kotlarski," Econometric Theory, 4, 925-932. [724]

Gallant, A. R., P. E. Rossi, AND G. TAUCHEN (1993): "Nonlinear Dynamic Structures,” Econometrica, 61 (4), 871-907. [718]

GeweKe, J., AND M. KeAne (2000): "An Empirical Analysis of Earnings Dynamics Among Men in the PSID: 1968-1989," Journal of Econometrics, 96 (2), 293-356. [697]

Golosov, M., AND A. TsYvinski (2015): "Policy Implications of Dynamic Public Finance," Annual Reviews of Economics, 7, 147-171. [693]

Golosov, M., M. Troshrin, AND A. Tsyvinski (2016): "Redistribution and Social Insurance," American Economic Review, 106 (2), 359-386. [693,694]

GOURINCHAS, P. O., AND J. A. PARKER (2002): “Consumption Over the Life Cycle,” Econometrica, 70, 47-91. [694]

Guvenen, F., And A. Smith (2014): "Inferring Labor Income Risk From Economic Choices: An Indirect Inference Approach,” Econometrica, 82 (6), 2085-2129. [694]

Guvenen, F., F. KARAHAn, S. OzCAN, AND J. SOng (2015): "What Do Data on Millions of U.S. Workers Reveal About Life-Cycle Earnings Risk?” Report, University of Minnesota. [697,715] 
GuVEnEn, F., S. OzCAN, AND J. SONG (2014): “The Nature of Countercyclical Income Risk," Journal of Political Economy, 122 (3), 621-660. [697,723]

HALL, R., AND F. MishKIN (1982): "The Sensitivity of Consumption to Transitory Income: Estimates From Panel Data of Households," Econometrica, 50 (2), 261-281. [694,707]

Heathcote, J., K. Storesletten, AND G. Violante (2014): “Consumption and Labor Supply With Partial Insurance: An Analytical Framework," American Economic Review, 104 (7), 2075-2126. [694]

Horowitz, J. L., AND M. MARKATOU (1996): "Semiparametric Estimation of Regression Models for Panel Data," Review of Economic Studies, 63, 145-168. [714]

Hu, Y. (2015): "Microeconomic Models With Latent Variables: Applications of Measurement Error Models in Empirical Industrial Organization and Labor Economics," Technical Report, Cemmap Working Paper CWP03/15. [724]

HU, Y., AND S. M. SCHENNACH (2008): "Instrumental Variable Treatment of Nonclassical Measurement Error Models,” Econometrica, 76, 195-216. [696,704,707,724-726]

HU, Y., AND J.-L. SHIU (2012): "Nonparametric Identification Using Instrumental Variables: Sufficient Conditions for Completeness," Unpublished Manuscript. [707]

HU, Y., AND M. SHUM (2012): "Nonparametric Identification of Dynamic Models With Unobserved State Variables," Journal of Econometrics, 171, 32-44. [697,704]

HUCKFELDT, C. (2016): "Understanding the Scarring Effect of Recessions," Report, Economics Department, Cornell University. [695]

HugGETT, M. (1993): "The Risk-Free Rate in Heterogeneous-Agent Incomplete-Insurance Economies," Journal of Economic Dynamics and Control, 17, 953-969. [695]

JAPPELLI, T., AND L. PISTAFERRI (2010): "The Consumption Response to Income Changes," Annual Reviews in Economics, 2, 479-506. [693]

KAPLAN, G., AND G. ViOlante (2010): "How Much Consumption Insurance Beyond Self-Insurance," American Economic Journal, 2 (4), 53-87. [694] 1199-1239. [694,704]

KIM, T. H., AND H. WHITE (2004): “On More Robust Estimation of Skewness and Kurtosis," Finance Research Letters, 1, 56-73. [699]

KOENKER, R., AND G. J. BASSETT (1978): “Regression Quantiles,” Econometrica, 46, 33-50. [726]

KOENKER, R., AND Z. XIAO (2006): “Quantile Autoregression,” Journal of the American Statistical Association, 101 (475), 980-990. [699]

Koop, G., M. H. Pesaran, AND S. M. PotTer (1996): "Impulse Response Analysis in Nonlinear Multivariate Models," Journal of Econometrics, 74 (1), 119-147. [718]

KRUEGER, D., K. MITMAN, AND F. PeRRI (2015): “Macroeconomics and Heterogeneity, Including Inequality,” Unpublished Manuscript. [707]

Krusell, P., AND A. SMith (1998): "Income and Wealth Heterogeneity in the Macroeconomy," Journal of Political Economy, 106, 867-896. [707]

Lise, J. (2013): “On-the-Job Search and Precautionary Savings,” Review of Economic Studies, 80 (3), 10861113. [695]

LOCHNER, L., AND Y. SHIN (2014): "Understanding Earnings Dynamics: Identifying and Estimating the Changing Roles of Unobserved Ability, Permanent and Transitory Shocks," Unpublished Manuscript. [696]

LOW, H. L., C. MEGHIR, AND L. PistAfERRI (2010): "Wage Risk and Employment Risk Over the Life-Cycle," American Economic Review, 100, 1432-1467. [723]

MEghiR, C., AND L. PISTAFERRI (2004): “Income Variance Dynamics and Heterogeneity,” Econometrica, 72 (1), 1-32. [695,715]

(2011): "Earnings, Consumption and Life Cycle Choices," in Handbook of Labor Economics, Vol. 4B. Amsterdam: Elsevier, 773-854. [694]

MISRA, K., AND P. SURICO (2014): "Consumption, Income Changes, and Heterogeneity: Evidence From Two Fiscal Stimulus Programs,” American Economic Journal: Macroeconomics, 6 (4), 84-106. [696]

NEWEY, W., AND J. POWELl (2003): "Instrumental Variable Estimation of Nonparametric Models," Econometrica, 71 (5), 1565-1578. [704]

NIELSEN, S. F. (2000): “The Stochastic EM Algorithm: Estimation and Asymptotic Results,” Bernoulli, 6 (3), 457-489. [711]

RIETZ, T. (1988): “The Equity Risk Premium: A Solution,” Journal of Monetary Economics, 22 (1), 117-131. [695]

SCHMIDT, L. (2015): “Climbing and Falling off the Ladder: Asset Pricing Implications of Labor Market Event Risk,” Unpublished Manuscript. [695] 
TERÄSVIRTA, T. (1994): "Specification, Estimation, and Evaluation of Smooth Transition Autoregressive Models," Journal of the American Statistical Association, 89, 208-218. [697]

WeI, Y., AND R. J. CARRoll (2009): “Quantile Regression With Measurement Error,” Journal of the American Statistical Association, 104, 1129-1143. [696,709]

Wilhelm, D. (2015): "Identification and Estimation of Nonparametric Panel Data Regressions With Measurement Error,” Cemmap Working Paper CWP34/15. [696,704,724]

CEMFI, Casado del Alisal, 5, Madrid 28014, Spain; arellano@cemfi.es, University College London, Gower Street, London WC1E 6BT, U.K. and Institute for Fiscal Studies, 7 Ridgmount Street, London WC1E 7AE, U.K.; r.blundell@ucl.ac.uk, and

Dept. of Economics, University of Chicago, 1126 East 59th Street, Chicago, IL 60637, U.S.A.; sbonhomme@uchicago.edu.

Co-editor Giovanni L. Violante handled this manuscript, which is based on a Presidential Address.

Manuscript received 8 September, 2015; final version accepted 30 January, 2017; available online 2 February, 2017. 